\title{
Nine Things Genomics Can Tell Us About Candida auris
}

\author{
Aleksandra D. Chybowska ${ }^{1}$, Delma S. Childers ${ }^{2}$ and Rhys A. Farrer ${ }^{3 *}$ \\ 'School of Medicine, Medical Sciences, and Nutrition, Institute of Medical Sciences, University of Aberdeen, Aberdeen, \\ United Kingdom, ${ }^{2}$ Aberdeen Fungal Group, Institute of Medical Sciences, University of Aberdeen, Aberdeen, \\ United Kingdom, ${ }^{3}$ Medical Research Council Centre for Medical Mycology at The University of Exeter, Exeter, \\ United Kingdom
}

\section{OPEN ACCESS}

Edited by:

Bridget Marie Barker,

Northern Arizona University,

United States

Reviewed by:

Anastasia Litvintseva,

Centers for Disease Control and Prevention (CDC), United States

Jolene Bowers,

Translational Genomics Research Institute, United States

*Correspondence:

Rhys A. Farrer rfarrer@broadinstitute.org;

r.farrer@exeter.ac.uk

Specialty section:

This article was submitted to Evolutionary and Genomic Microbiology,

a section of the journal

Frontiers in Genetics

Received: 14 January 2020

Accepted: 23 March 2020

Published: 15 April 2020

Citation:

Chybowska AD, Childers DS and Farrer RA (2020) Nine Things

Genomics Can Tell Us About Candida

auris. Front. Genet. 11:351.

doi: 10.3389/fgene.2020.00351
Candida auris is a recently emerged multidrug-resistant fungal pathogen causing severe illness in hospitalized patients. C. auris is most closely related to a few environmental or rarely observed but cosmopolitan Candida species. However, C. auris is unique in the concern it is generating among public health agencies for its rapid emergence, difficulty to treat, and the likelihood for further and more extensive outbreaks and spread. To date, five geographically distributed and genetically divergent lineages have been identified, none of which includes isolates that were collected prior to 1996. Indeed, C. auris' ecological niche(s) and emergence remain enigmatic, although a number of hypotheses have been proposed. Recent genomic and transcriptomic work has also identified a variety of gene and chromosomal features that may have conferred $C$. auris with several important clinical phenotypes including its drug-resistance and growth at high temperatures. In this review we discuss nine major lines of enquiry into $C$. auris that big-data technologies and analytical approaches are beginning to answer.

\footnotetext{
Keywords: Candida auris, genomics, emergence, antifungal resistance mechanisms, virulence factors, epigenetics
}

\section{THE EVOLUTION OF C. auris AND CLOSEST RELATIVES}

Candida auris was first isolated from the external ear canal (auris is Latin for "ear") of an 70 years old Japanese woman, who was a patient at Tokyo Metropolitan Geriatric Hospital (Tokyo, Japan) in 2009 (Satoh et al., 2009). This first isolate (type strain JCM15448; CBS10913; DSM21092) had several attributes that distinguished it from its closest known relatives, warranting its demarcation as a new species. Unique features of $C$. auris included the ability to grow (albeit slow and with weak growth) at $42^{\circ} \mathrm{C}$, while isolates from its three closest relatives (C. haemulonii, C. pseudohaemulonii, or C. heveicola) were unable to grow at this temperature (Satoh et al., 2009). JCM15448 showed a reduced ability to assimilate different carbon sources (galactose, 1 -sorbose, cellobiose, 1 -arabinose, ethanol, glycerol, salicin, or citrate), whilst $C$. ruelliae was able to assimilate all of those as carbon sources (although C. ruelliae is able to grow at $42^{\circ} \mathrm{C}$ ). JCM15448 produced no pseudohypae while all closest relatives (C. haemulonii species complex. C. heveicola, and C. ruelliea) were able to. The authors concluded that isolate JCM15448 belonged to a new species, and "may have pathogenicity, but this will be elucidated in future studies" (Satoh et al., 2009).

C. auris falls within the Clavispora clade of the Metschnikowiaceae family of the order Saccharomycetales, which are ascomycetous (hemiascomycetes) yeasts that reproduce by budding. 


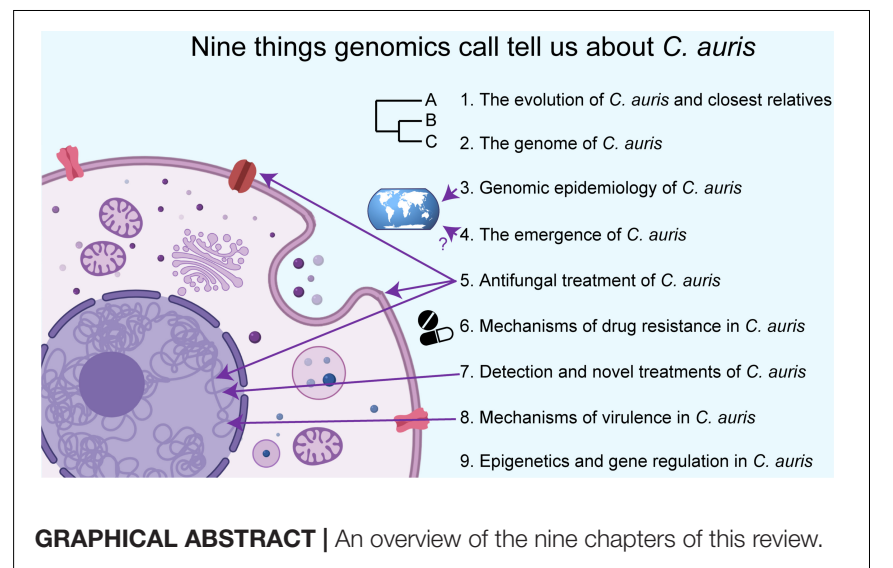

Saccharomycetales includes approximately 1000 described species including human commensals and pathogens (e.g., C. albicans), plant pathogens (e.g., Eremothecium gossypii), species important for baking and brewing (e.g., Saccharomyces cerevisiae), species that have associations and interactions with plants and/or arthropods, as well as numerous free living saprobes. The most widely studied Candida species are C. albicans, C. tropicalis, and C. parapsilosis from the CTG clade - defined by predominantly translating the codon CTG to serine instead of leucine (Santos et al., 2011). Genetically distinct is C. glabrata, which falls in the Nakaseomyces clade (Angoulvant et al., 2016) and is also of growing interest primarily due to its increasing prevalence in nosocomial infections (Toda et al., 2019). These four species together accounted for approximately $90 \%$ of species responsible for invasive candidiasis during 2003 (Pfaller and Diekema, 2007). Although C. auris is genetically divergent from the other commonly studied CTG clade species, it also translates the codon CTG to serine and is within the CTG clade (Muñoz et al., 2018). C. auris' genetic distance from other more commonly studied species and its recent emergence raises many questions regarding its evolution, epidemiology, and the genetic basis for its pathogenicity and drug resistance.

The phylogenetic relationship of C. auris to other known species is still not fully resolved, mainly owing to the rarity of some of its closest relatives. Maximum likelihood phylogenetic reconstruction has revealed that four of the five clades of C. auris (with genomic analyses of Clade $\mathrm{V}$ not reported yet) are highly genetically related $(98.7 \%$ average pairwise nucleotide identity), all of which are more distantly related to other Candida species that have their genome sequenced including C. haemulonii, C. duobushaemulonii, and C. psuedohaemulonii (88\% average pairwise nucleotide identity) (Muñoz et al., 2018). Given the recency of emergence and discovery of already five clades within the C. auris species, it seems likely that further clades await discovery. Muñoz et al. found the most closely related species to be C. haemulonii, followed by C. pseudohaemulonii - both of which are occasionally found in animal or clinical settings (Cendejas-Bueno et al., 2012).
Early non-whole-genome sequencing (specifically 26S rDNA D1/D2 domain) suggested C. auris may be more closely related to C. ruelliae than C. haemulonii (Satoh et al., 2009), a species identified from the flowers of the Ruellia species of the Acanthaceae family (Saluja and Prasad, 2008). C. ruelliae has not been reported since to the best of our knowledge, and has not had its genome sequenced, thereby hampering efforts to phylogenetically place it, and verify its relationship to $C$. auris. ITS and rDNA sequencing meanwhile suggest that $C$. auris groups phylogenetically with C. heveicola (Satoh et al., 2009), a species described by a single isolate YJ2E(T) identified from tree sap from tropical forests in Yunnan and Hainan Provinces in southern China (Wang et al., 2008). Similarly, C. heveicola has also not been re-identified or had its full genome sequenced. Evidently, much remains to be known about true genome diversity of both C. auris and its closest relatives.

One of the closest known relatives of C. auris is C. haemulonii, which was first discovered in 1962 (formerly named Torulopsis haemulonii) from the gut of a blue-striped grunt fish (Haemulon scirus) (van Uden and Kolipinski, 1962), which is found in the Western Atlantic, Gulf of Mexico and the Caribbean. It was also isolated from the skin of dolphins and seawater off the coast of Portugal (van Uden and Kolipinski, 1962; Khan et al., 2007). C. haemulonii has also been found from terrestrial sources including the roots of Cassava (Manihot esculenta) in Brazil in 2010 , and in a laboratory tick colony (Ornithodoros moubata) in the Czech Republic in 2001 (Jackson et al., 2019). C. haemulonii has also been the cause of candidemia and other bloodstream infections, catheter-related fungemia, osteitis, and even outbreaks in intensive care units (Cendejas-Bueno et al., 2012) including in Kuwait in 2005 (Khan et al., 2007).

In 1993, Lehmann et al. (1993) found C. haemulonii to comprise two separate groups (group I and II). In 2012, CendejasBueno et al. (2012) redefined group I as the C. haemulonii species, and re-categorized group II as the new species C. duobushaemulonii based on genetic differences, ability to grow at different temperatures and on different carbon sources, and drug susceptibilities. C. duobushaemulonii has been isolated from patients' blood and foot ulcers in Asia, Europe, and North America, and has been the cause of recurrent vulvovaginal candidiasis (VVC) in Brazil (Jackson et al., 2019). C. duobushaemulonii has also been isolated from a firebug (Pyrrhocoris apterus) in Germany (Jackson et al., 2019). In 2006, Sugita et al. (2006) described a closely related but distinct species to C. haemulonii, C. pseudohaemulonii, which was isolated from the blood of a Thai patient. Recent genome sequencing showed $92 \%$ nucleotide genome identity between C. pseudohaemulonii and C. duobushaemulonii and several chromosomal rearrangements confirming their genetic divergence (Muñoz et al., 2018).

The evolution and phylogenetics of C. auris remain enigmatic, due to the disparate ecological sources of its closest relatives, their genetic distance, and that most have not yet had their genomes sequenced and compared, thus precluding detailed population genetic analysis within these species. The discovery of more closely related species by concerted sampling efforts, along with genome sequencing of others already identified but without 
reference genomes such as C. ruelliae or C. heveicola may provide additional clues about their and $C$. auris' evolution.

\section{THE GENOME OF C. auris}

To date, five genetically diverse clades have been discovered. The first genome assemblies came from isolates belonging to Clade I, such as the Illumina-based genome of isolate Ci6684 (Chatterjee et al., 2015), and Illumina and Nanopore based genomes of five isolates from an outbreak in London, United Kingdom (Rhodes et al., 2018b). Shortly after, Muñoz et al. (2018) assembled and compared genomes across four (Clade I- > IV) of the then known lineages. Muñoz mapped the genomes into seven chromosomes using optical maps, and recent work using long-reads and telomere-to-telomere assemblies confirm the chromosome count. Genome assembly sizes ranged from 12.1 and $12.7 \mathrm{Mb}$, and the number of predicted protein coding genes ranged from 5,288 and 5,601, similar to other Candida species. The correlation between the genome assembly size and the number of predicted genes was surprisingly negative $(n=7$, $r^{2}=0.0725$ ) (Muñoz et al., 2018). To date, the genome of clade $\mathrm{V}$ has not been reported.

The seven chromosomes of C. auris have undergone chromosomal rearrangements between clades. For example, inversions and translocations of hundreds of kilobase genomic regions have been reported between Clade I (isolate B8441) and Clade III (isolate B11221) (Muñoz et al., 2018). More recent work using long-read telomere-to-telomere sequencing has shown the Clade II genome appears highly rearranged compared with the other 3 clades, with 2 inversions and 9 translocations resulting in a substantially different karyotype (Muñoz et al., 2019). It was hypothesized that these rearrangements could potentially prevent genetic exchange between those lineages (Muñoz et al., 2018). However, a phylogenetically conserved (at least between C. auris, C. haemulonii species complex, and C. lusitaniae) mating type locus (MTL) is present in the C. auris genome, consisting of a1 and a2 genes in MTLa isolates and a single $\alpha 1$ gene in the MTL $\alpha$ isolates (Muñoz et al., 2018). Interestingly, Clade I and Clade IV of $C$. auris isolates have so far all been MTLa, while Clade II and Clade III have all been MTL $\alpha$. The mating type of Clade V has not yet been reported. The presence of a conserved mating type suggests that $C$. auris can mate, despite a lack of clear recombinants between or within clades.

Genomic variation between and within clades of $C$. auris may have an impact on gene function. The four clades of C. auris share an average pairwise nucleotide identity of $98.7 \%$ (Muñoz et al., 2018). In terms of nucleotide diversity $(\pi$ ), the four clades of $C$. auris had a value of 0.0039 which is less than C. neoformans var. grubii and more than the exclusively clonal Trichophyton rubrum (Muñoz et al., 2018). In terms of microevolution, Chow et al. (2018) found between 0 and 12 SNPs among clinical and screening cases in the United States within a patient, and also similar numbers within the facility during an outbreak. Comparisons between C. auris and its closest relatives showed changes to genes linked to drug resistance and virulence, including expanded families of transporters and lipases (Muñoz et al., 2018). However, much work remains to study positive selection between clades, and various features of population genetics within clades - including the identification of crossovers, effective population sizes, fixation indices, or evidence for bottlenecking.

Given the rapid clinical emergence of $C$. auris, including the newly identified Clade V in 2018 (Chow et al., 2019), the allelic variation within the species remains unclear. Further sampling of C. auris is clearly necessary, both in terms of screening historic samples that may have been mischaracterized, as well as new sampling efforts in both clinical and environmental settings. The discovery of additional clades, and the identification of clades with multiple mating types would assist in understanding the evolution of $C$. auris. A greater understanding of $C$. auris' phylogenetics and population genetics would also be achieved by additional bioinformatic analysis - including a comprehensive characterization of gene gain/loss events and selection of alleles between or within those species or clades.

\section{GENOMIC EPIDEMIOLOGY OF C. auris}

Since 2009, five genetically diverse clades have been discovered from India and Pakistan (South Asian; Clade I), Japan (East Asian; Clade II), South Africa (African; Clade III), Venezuela (South American; Clade IV) (Lockhart et al., 2017b) and most recently in 2019, Iran (Clade V) (Chow et al., 2019). Over the past decade, isolates of $C$. auris have also been detected across all major continents, including elsewhere in Asia, Europe, the Middle East, Africa, Australia, and North and South America (Table 1). This nearly simultaneous and recent independent emergence is both unprecedented and raises questions regarding its origins and transmission events.

Genome sequencing of isolates from clinical environments is revealing detailed insights into how C. auris is spreading between continents, within countries, and even within wards of hospitals and over time in patients. For example, between 2013 and 2017, 133 isolates (73 clinical cases and 60 screening cases) from ten US states were collected and evaluated by whole genome sequencing (Chow et al., 2018) and compared to standardized case report forms and contact investigations, including travel history and epidemiological links. Chow et al. found that isolates were related to South Asian, South American, African and East Asian isolates, indicating multiple clades of C. auris were introduced into the United States, some of which perhaps several times. Surprisingly however, only $7 \%$ of the clinical cases had clear evidence of being acquired through health-care exposures abroad, suggesting travel data were lacking, the index patient with exposure were not identified, or that the infection many may have been acquired from other sources, perhaps including environmental sources. Chow et al. found a similarly small number of SNPs (between 0 and 12) among clinical and screening cases within a patient compared with those within a facility during an outbreak. The low numbers of genomic variants indicate that there is local and ongoing transmission of C. auris in the United States, with most (82\%) of the clinical isolates reported from hospitals 
TABLE 1 | C. auris has caused outbreaks across all major continents over the last decade.

\begin{tabular}{|c|c|c|c|c|c|}
\hline Region & Country & Details & Clade(s) & Date & Citation \\
\hline \multirow[t]{7}{*}{ Asia } & China & $\begin{array}{l}15 \text { patients, } 35 \text { isolates ( } 26 \text { from urine, } 4 \text { catheter, } 3 \\
\text { sputum, } 1 \text { blood, } 1 \text { fluid) }\end{array}$ & III & $2011-2017$ & Tian et al., 2018 \\
\hline & India & 3 hospitals, 19 isolates & । & $2012-2015$ & Lockhart et al., 2017b \\
\hline & Japan & 1 patient (external ear canal) & $\|$ & 2009 & Satoh et al., 2009 \\
\hline & Malaysia & 1 hospital, 1 patient & Unknown & 2017 & Mohd Tap et al., 2018 \\
\hline & Pakistan & 2 hospitals, 18 isolates & 1 & $2010-2015$ & Lockhart et al., 2017b \\
\hline & Singapore & $\begin{array}{l}1 \text { hospital, } 3 \text { patients (all with recent hospitalizations in India } \\
\text { or Bangladesh) }\end{array}$ & Unknown & 2012-2017 & Tan and Tan, 2018 \\
\hline & South Korea & 61 patients (4 blood, 57 ear) from 13 hospitals & $\|$ & $1996-2018$ & Kwon et al., 2019 \\
\hline \multirow[t]{10}{*}{ Europe } & Austria & 1 case & Unknown & 2018 & Kohlenberg et al., 2018 \\
\hline & Belgium & 1 case & Unknown & 2013-2017 & Kohlenberg et al., 2018 \\
\hline & France & 2 cases & Unknown & $2013-2017$ & Kohlenberg et al., 2018 \\
\hline & Germany & 7 cases (6 from patients previously hospitalized abroad) & ।, III & 2015-2017 & Hamprecht et al., 2019 \\
\hline & Norway & 1 case & Unknown & $2013-2017$ & Kohlenberg et al., 2018 \\
\hline & Russia & 1 hospital (49 cases in an ICU) & 1 & 2016-2017 & Barantsevich et al., 2019 \\
\hline & Spain & 79 isolates, 738 environmental samples & Unknown & 2017-2019 & Ruiz-Gaitán et al., 2019 \\
\hline & Switzerland & $\begin{array}{l}1 \text { patient who was on holiday in Spain, transferred to } \\
\text { Switzerland hospital }\end{array}$ & Unknown & 2017 & Riat et al., 2018 \\
\hline & The Netherlands & 2 cases (both from patients previously hospitalized in India) & 1 & 2019 & Vogelzang et al., 2019 \\
\hline & United Kingdom & 72 patients (colonization, candidaemia, vascular lines) & I & $2015-2016$ & Rhodes et al., 2018b \\
\hline \multirow[t]{6}{*}{ Middle East } & Iran & 1 patient & V & 2018 & Chow et al., 2019 \\
\hline & Israel & 2 hospitals, 5 patients & Unknown & 2014-2015 & Ben-Ami et al., 2017 \\
\hline & Kuwait & 56 patients, 158 isolates & Unknown & $2014-2017$ & Khan et al., 2018 \\
\hline & Oman & 2 patients & Unknown & $2016-2017$ & Mohsin et al., 2017 \\
\hline & Saudi Arabia & 1 hospital, 2 patients & 1 & 2017-2018 & Abdalhamid et al., 2018 \\
\hline & United Arab Emirates & 1 hospital, 1 patient & Unknown & 2017 & Alatoom et al., 2018 \\
\hline \multirow[t]{2}{*}{ Africa } & Kenya & 1 patient & III & 2012 & Heath et al., 2019 \\
\hline & South Africa & 1,576 cases & III & 2012-2016 & Govender et al., 2018 \\
\hline Australia & Australia & 1 patient (a 65-year old with recent hospitalization in Kenya) & III & 2015 & Heath et al., 2019 \\
\hline \multirow[t]{5}{*}{ Americas } & Canada & 1 patient (a 64-year old with a recent hospitalization in India) & Unknown & 2017 & Schwartz and Hammond, 2017 \\
\hline & Columbia & 4 hospitals, 7 people colonized, 37 environmental samples & IV & 2015-2016 & Escandón et al., 2019 \\
\hline & Panama & 1 hospital, 9 patients, 14 isolates & Unknown & 2016 & Araúz et al., 2018 \\
\hline & United States & $\begin{array}{l}10 \text { US states ( } 133 \text { isolates; } 73 \text { clinical cases and } 60 \\
\text { screening cases) }\end{array}$ & I, II, III, IV & 2013-2017 & Chow et al., 2018 \\
\hline & Venezuela & 1 hospital, 5 isolates & IV & 2012-2013 & Lockhart et al., 2017b \\
\hline
\end{tabular}

located in New York and New Jersey (Chow et al., 2018; Tracking Candida auris | CDC, 2020).

Outbreaks in Europe and Australia have also been attributed to recent spread from other continents. For example, of the 7 cases of C. auris identified in Germany during 2015-2017, 6 were from patients previously hospitalized abroad (Hamprecht et al., 2019). Whole-genome sequencing and epidemiologic analyses revealed that all patients in Germany were infected with different strains: five related to isolates from South Asia (Clade I), and one related to isolates from South Africa (Clade III) (Hamprecht et al., 2019). More recently (2019), the first two cases of $C$. auris were reported in The Netherlands, with both cases arising in patients that were treated in a healthcare facility in India prior to admission. Indeed, genetic analysis showed that these isolates also belonged to the South Asian C. auris Clade I (Vogelzang et al., 2019). In Australia in 2015, a 65-year-old man with a history of intensive care treatment in Kenya in 2012, was diagnosed with the
South African Clade III of C. auris causing sternal osteomyelitis (Heath et al., 2019).

The largest outbreak in the United Kingdom to date of C. auris occurred between April 2015 and November 2016 in the Royal Brompton hospital in London, which involved 72 separate patients (Schelenz et al., 2016). Using Oxford Nanopore and Illumina sequencing technologies to gather genomic data on those clinical isolates, Rhodes et al. placed the United Kingdom outbreak in the India/Pakistan clade (Clade I), demonstrating an Asian origin for the outbreak. Rhodes et al. (2018a) were also able to estimate the timing for the most recent common ancestor (MRCA) of those outbreak strains to late March 2015, which was just weeks prior to the first patient identified with a C. auris infection. Additionally, by using root-to-tip regression, Rhodes et al. (2018a) estimated the evolutionary rate of the C. auris nuclear genome to $5.7 \times 10^{-5}$ substitutions per site per year, a slower rate to the nuclear DNA of other related fungal species in the Saccharomycetales such as Saccharomyces cerevisiae 
$\left(5.7 \times 10^{-3}\right)$, as well as more distantly related species such as the Schizosaccharomyces pombe traditional beer strains $\left(3.0 \times 10^{-3}\right)$.

Multiple outbreaks of C. auris in Colombia between 2015 and 2016 led to a concerted effort to understand its epidemiology within Colombian health-care clinics (Escandón et al., 2019). By sampling infected patients, patient contacts, healthcare workers, and the environment across 4 hospitals, they identified likely sources of transmission events, including health-care workers, and in the hospital environment. For all studied groups, the swabbed body sites included: axillae, groins, nostrils and ears. Samples were also taken from the mouth and rectum of patients. Among patients, the axilla, groin, and rectum had the highest positivity rate $(2 / 7 ; 28 \%$ each). C. auris was isolated from the hands of two healthcare workers and the groin of another healthcare worker (Escandón et al., 2019). None of the 4 patient contacts swabbed were positive for $C$. auris, although the low sample size does not preclude this as a mechanism of spread. C. auris was isolated from 37 of 322 (11\%) environmental samples, including a diverse range of hospital equipment including on a stethoscope, hospital floor, body cables, bed railing, mattresses, stretchers, bedpans, a closet, towel, cell phone, mops, TV control, meal table, sink, toilet, wheelchair, and a nurse's shoe. The results demonstrate that contamination can occur, and also that $C$. auris can survive on a variety of substances, at least in the short term (Escandón et al., 2019). Unlike the aforementioned European outbreaks, whole-genome sequencing and phylogenomics showed that the genetic background of isolates throughout the four hospitals were all similar (suspected Clade IV), despite the four hospitals spanning $700 \mathrm{~km}$ across Colombia. Isolates from the two northern Colombian hospitals grouped into a sub-clade, while those from the two southern hospitals grouped into a separate sub-clade, suggesting some geographically defined population structure in the country, and possibly endemism.

Since it was first discovered in Tokyo Metropolitan Geriatric Hospital (Tokyo, Japan) in 2009 (Satoh et al., 2009), C. auris has caused hospital acquired infections and outbreaks across all main continents including Asia, Europe, Africa, Australia, North America and South America. Given the recency of these outbreaks, further outbreaks across the world in the upcoming decade appear likely, unless concerted mitigation efforts are made (Escandón et al., 2019; Ong et al., 2019). It is also likely that cases are being misidentified (Chatterjee et al., 2015; Govender et al., 2018) or undetected, especially across SubSaharan Africa, South America and Eastern Europe where there are currently few reports (Ong et al., 2019). Exemplifying this is how common $C$. auris has become in some hospitals from regions with few additional reports of cases (van Schalkwyk et al., 2019). For example, in the Aga Khan University Hospital in Nairobi, Kenya between 2010 and 2016, C. auris was the most common cause of candidemia (38\% of 201 patients) (Adam et al., 2019), suggesting that other hospitals in Kenya and in neighboring countries are likely to also harbor C. auris. Necessary steps to better understanding the epidemiology of C. auris will therefore include greater sampling and reporting from regions that underreported $C$. auris cases, a transition away from unreliable diagnostic methods, and further genomic analysis describing the population genetics of the various lineages, all of which may reveal its geospatial origin and subsequent spread.

\section{THE EMERGENCE OF C. auris}

To date, the earliest reports of $C$. auris have been from a Clade II isolate in 1996, retrospectively from a case of misidentified nosocomial fungemia in South Korea (Lee et al., 2011). The species was first described over 10 years later from a case in Japan (Satoh et al., 2009), and simultaneously arising in South Africa with a Clade III isolate also from 2009 (Govender et al., 2018). C. auris has not been detected earlier than 2009 by The SENTRY Antifungal Surveillance Program, which has been collecting consecutive invasive Candida isolates from 135 participating medical centers in North America, Europe, Latin America and the Asia-Pacific regions since 1997 (Pfaller et al., 2019). However, molecular clock estimates from 304 worldwide genomes date the most recent common ancestor of each of the four clades within the last 339 years, and outbreak isolates from Clades I, III, and IV to 34-35 years ago ( $~ 10$ years prior to first being detected) (Chow et al., 2020). While the details of its origin remain unclear, $C$. auris is not the only fungal pathogen to have recently clinically emerged. Indeed, a variety of phylogenetically diverse emerging fungal pathogens are rapidly increasing in their incidence, geographic or host range and virulence (Morse, 1995; Farrer and Fisher, 2017). Recently highlighted examples include the emergence and increasing incidence of a separate ascomycete responsible for human infections: Emergomyces africanus (Dukik et al., 2017), the newly-described chytrid fungus Batrachochytrium salamandrivorans causing rapid declines of fire salamanders across an expanding region of northern Europe (Farrer, 2019), and the basidiomycete fungus Cryptococcus gattii expanding its range into non-endemic environments with a consequential increase of fatal disease in humans (Fraser et al., 2005; Byrnes et al., 2010).

One suggestion for the absence of $C$. auris isolates prior to 1996 is that it has only recently become part of the human microbiome, and has either recently acquired sufficient virulence to cause invasive infections or been newly introduced into human populations in which invasive Candida infections can be identified (Jackson et al., 2019). For example, recent genetic recombination, hybridization, or other biological changes could potentially increase the organism's transmissibility or virulence (Jackson et al., 2019). However, for this hypothesis to hold, such genetic changes would need to have occurred across all clades of C. auris recently.

One line of inquiry to understand the recency of the emergence has been to re-screen isolates taken prior to 2009 that may have been misdiagnosed as other Candida species. Indeed, $C$. auris has been misdiagnosed as its closest relatives C. haemulonii or C. pseudohaemulonii (Chatterjee et al., 2015), species which have even been identified alongside $C$. auris in the same month in the same hospital (Ben-Ami et al., 2017). Lee et al. (2011) identified the oldest known case of $C$. auris from South Korea, which dates to 1996, belongs to Clade II, and had 
been misidentified as $C$. haemulonii by Vitek 2. Another clinical isolate originally characterized as C. haemulonii from a hospital in South Africa, was later shown to be a Clade III isolate of C. auris (Govender et al., 2018). This isolate was taken from a patient in 2009 demonstrating independent emergences in 2009 of at least two of the C. auris lineages (Clade II and Clade III). A recent study comparing 204 genomes of $C$. auris using Bayesian molecular clock phylogenetics estimated the origin of each of the four clades within the last 339 years, and outbreak clusters (Clade I, III, and IV) originating 34-35 years ago (Chow et al., 2020), suggesting more historic samples were not identified.

The spread of $C$. auris within and between hospital settings has been clearly demonstrated by at least clades I, III and IV (e.g., Chow et al., 2018; Rhodes et al., 2018b; Escandón et al., 2019), so it is possible that abiotic factors could explain the emergence. For example, human activities such as deforestation, expansion of farmland, and coastal ecosystem disruption could have allowed an ecological jump (Jackson et al., 2019). Indeed, C. auris' closest relatives include C. ruelliae from flowers of the Ruellia species (Saluja and Prasad, 2008), and C. haemulonii that can also cause clinical infections but also found in the GI tract and skin of marine animals (van Uden and Kolipinski, 1962; Khan et al., 2007). Furthermore, C. auris has been shown to persist on a wide variety of hospital equipment (Escandón et al., 2019). It therefore seems likely that $C$. auris may also have an environmental niche (plants or aquatic). The widespread use of fungicides, especially triazoles in agriculture could have been responsible for C. auris to become more prominent in the environment, as it shows high levels of antifungal resistance. At the same time, increased human travel, trade or other anthropogenic factors may have simply led to the globalization and emergence of $C$. auris (Jackson et al., 2019).

C. auris is able to grow (albeit slowly) at $42^{\circ} \mathrm{C}$, while isolates from close relatives (C. haemulonii, C. pseudohaemulonii or C. heveicola) were unable to grow at this temperature (Satoh et al., 2009). C. auris' thermotolerance enables it to cause invasive candidemia, including tolerating the fever response (Jackson et al., 2019), but may also enable it to sustain body temperatures of other animals including birds (Casadevall et al., 2019). For example, pigeons body temperature can be up to $44^{\circ} \mathrm{C}$ during flight (Aulie, 1971), compared with only a few degrees above $37^{\circ} \mathrm{C}$ during exercise for humans (Gleeson, 1998). Similarly, sea birds may serve as reservoirs for indirect transmission of drug-resistant Candida species, such as C. glabrata, to humans (Al-Yasiri et al., 2016; Casadevall et al., 2019). C. auris is also able to survive high salt concentrations (broth containing $10 \% \mathrm{NaCl}$ ), which might allow it survive environmentally including hypersaline desert lakes, salt-evaporating ponds, or tidal pools (Jackson et al., 2019). Thus, C. auris is thought likely to survive both in the environment, as well as colonizing human skin. On skin it is likely to compete with other commensals such as Malassezia, although studies on $C$. auris interactions with bacteria or other fungi are lacking.

The thermotolerance of $C$. auris has led to the hypothesis that its emergence may be linked to climate change and global temperature changes, and may even be the first example of a new pathogenic fungus emerging from human-induced global warming (Casadevall et al., 2019). Indeed, it has been proposed that as the gap between the environmental temperature and human body temperature narrows, new fungal diseases of mammals will increase (Garcia-Solache and Casadevall, 2010). Casadevall et al. (2019) suggest a number of factors that supports the hypothesis of a climate change emergence, including that C. auris can grow on skin but not anaerobically in the gut (suggesting a recent environmental source), the constitutive overexpression of heat shock protein HSP90, and the inability of many relatives to grow at high temperatures. C. auris clades I, II, III, and IV differ by an average of $1.3 \%$ average pairwise nucleotide identity (Muñoz et al., 2018). Although a time to most recent common ancestor of multiple clades has not yet been predicted, the ancestor to all clades almost certainly predates industrial era warming, which begun in 1800 AD (Abram et al., 2016). Given that all known clades of $C$. auris are able to colonize skin and cause infections, its ability to colonize these niches are more likely to be an evolutionary conserved trait, rather than five independent recent trait acquisitions. It nevertheless remains to be shown if climate change had a role to play in exposing each clade into an alternative niche they already had the ability to colonize (Casadevall et al., 2019).

Further environmental sampling, genome sequencing and metagenomics studies focusing on fungi are necessary to identify and confirm environmental sources of $C$. auris. Further genomic epidemiology studies, including on the genetic diversity within each clade will help to find older natural populations that could indicate the progenitor of C. auris. Clade II (East Asian clade) exhibits higher genetic diversity than the other clades (Jackson et al., 2019), although it also exhibits large karyotypic variation and loss of sub-telomeric regions, suggesting it is not ancestral to Clade I, III and IV. The discovery of further ecological niches and the geographical origin will be important to prevent similar emergences in future as well as identify and mitigate emergence of new clades of $C$. auris that have the potential to cause outbreaks.

\section{ANTIFUNGAL TREATMENT OF C. auris}

Treating invasive fungal infections is a considerable challenge due to the limited number of available antifungal agents. The five major antifungal drug classes that are used in hospital settings are azoles, allylamines, polyenes, echinocandins and nucleoside analogs (Bidaud et al., 2018). To date, there are only a few alternative antifungal agents (de Oliveira Santos et al., 2018). While the efficacy of some antifungal classes is highly dependent on the site of infection due to their tissue penetration properties (Felton et al., 2014), other classes of antifungals (i.e., polyenes) often lead to serious side effects such as renal injury or cardiomyopathy (Bandeira et al., 2016), primarily due to conserved or structural similarities in drug targets such as cholesterol and ergosterol (Kamiñski, 2014). Given the lack of treatment options, the emergence of fungal resistance to even one of the major drug classes is very alarming, as it makes the infection considerably more challenging to treat. 
TABLE 2 | Candida auris resistance patterns.

\begin{tabular}{|c|c|c|c|c|c|c|}
\hline Drug category & Drug & Method & Resistant & $\begin{array}{c}\text { Samples } \\
\text { no }\end{array}$ & $\begin{array}{c}\text { Resistance threshold } \\
\text { used }(\mu \mathrm{g} / \mathrm{mL})\end{array}$ & References \\
\hline \multirow[t]{20}{*}{ Azoles } & Fluconazole & CLSI & $93 \%$ & 54 & 32 & Lockhart et al., 2017b \\
\hline & & CLSI & $86 \%$ & 123 & 32 & Arendrup et al., 2017 \\
\hline & & EUCAST & $96 \%$ & 123 & 32 & Arendrup et al., 2017 \\
\hline & & CLSI & $90 \%$ & 320 & 32 & Chowdhary et al., 2018 \\
\hline & Voriconazole & CLSI & $54 \%$ & 54 & 2 & Lockhart et al., 2017b \\
\hline & & CLSI & $33 \%$ & 123 & 2 & Arendrup et al., 2017 \\
\hline & & EUCAST & $15 \%$ & 123 & 2 & Arendrup et al., 2017 \\
\hline & & CLSI & $39 \%$ & 90 & 2 & Kathuria et al., 2015 \\
\hline & & Vitek 2 & $29 \%$ & 90 & 2 & Kathuria et al., 2015 \\
\hline & & Etest & $28 \%$ & 90 & 2 & Kathuria et al., 2015 \\
\hline & & CLSI & $15 \%$ & 320 & 2 & Chowdhary et al., 2018 \\
\hline & Isavuconazole & CLSI & $4 \%$ & 123 & 2 & Arendrup et al., 2017 \\
\hline & & EUCAST & $4 \%$ & 123 & 2 & Arendrup et al., 2017 \\
\hline & Itraconazole & CLSI & $0 \%$ & 54 & 2 & Lockhart et al., 2017b \\
\hline & & CLSI & $1 \%$ & 123 & 2 & Arendrup et al., 2017 \\
\hline & & EUCAST & $0 \%$ & 123 & 2 & Arendrup et al., 2017 \\
\hline & & CLSI & $6 \%$ & 320 & 1 & Chowdhary et al., 2018 \\
\hline & Posaconazole & CLSI & $0 \%$ & 54 & 2 & Lockhart et al., 2017b \\
\hline & & CLSI & $2 \%$ & 123 & 2 & Arendrup et al., 2017 \\
\hline & & EUCAST & $0 \%$ & 123 & 2 & Arendrup et al., 2017 \\
\hline \multirow[t]{8}{*}{ Polyenes } & Amphotericin B & CLSI & $35 \%$ & 54 & 2 & Lockhart et al., 2017b \\
\hline & & CLSI & $16 \%$ & 90 & 2 & Kathuria et al., 2015 \\
\hline & & Vitek 2 & $100 \%$ & 90 & 2 & Kathuria et al., 2015 \\
\hline & & Etest & $1 \%$ & 90 & 2 & Kathuria et al., 2015 \\
\hline & & CLSI & $8 \%$ & 320 & 2 & Chowdhary et al., 2018 \\
\hline & & CLSI & $10 \%$ & 123 & 2 & Arendrup et al., 2017 \\
\hline & & EUCAST & $0 \%$ & 123 & 2 & Arendrup et al., 2017 \\
\hline & Nystatin & CLSI & $100 \%$ & 320 & 2 & Chowdhary et al., 2018 \\
\hline \multirow[t]{13}{*}{ Echinocandins } & Anidulafungin & CLSI & $2 \%$ & 320 & 8 & Chowdhary et al., 2018 \\
\hline & & CLSI & $6 \%$ & 123 & 8 & Arendrup et al., 2017 \\
\hline & & EUCAST & $0 \%$ & 123 & 8 & Arendrup et al., 2017 \\
\hline & & CLSI & $7 \%$ & 54 & 8 & Lockhart et al., 2017b \\
\hline & Caspofungin & CLSI & $2 \%$ & 320 & 8 & Chowdhary et al., 2018 \\
\hline & & CLSI & $3 \%$ & 90 & 8 & Kathuria et al., 2015 \\
\hline & & Vitek 2 & $0 \%$ & 90 & 8 & Kathuria et al., 2015 \\
\hline & & Etest & $0 \%$ & 90 & 8 & Kathuria et al., 2015 \\
\hline & & CLSI & $7 \%$ & 54 & 8 & Lockhart et al., 2017b \\
\hline & Micafungin & CLSI & $2 \%$ & 320 & 8 & Chowdhary et al., 2018 \\
\hline & & CLSI & $7 \%$ & 54 & 8 & Lockhart et al., 2017b \\
\hline & & CLSI & $6 \%$ & 123 & 8 & Arendrup et al., 2017 \\
\hline & & EUCAST & $6 \%$ & 123 & 8 & Arendrup et al., 2017 \\
\hline Nucleoside analogs & 5-Flucytosine & CLSI & $6 \%$ & 54 & 128 & Lockhart et al., 2017b \\
\hline Allylamines & Terbinafine & CLSI & $100 \%$ & 320 & 2 & Chowdhary et al., 2018 \\
\hline
\end{tabular}

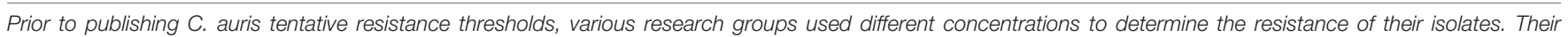
choices were based on a comparison of the treatment outcomes associated with drug exposure observed in C. auris and in other Candida spp. infections.

It is of considerable concern to health authorities that the emergent $C$. auris includes isolates that are resistant to all antifungals (Warris, 2018). C. auris isolates are resistant to fluconazole, the major drug in treating candidemia, with MIC values $>64 \mu \mathrm{g} / \mathrm{mL}$ (Chowdhary et al., 2018; Warris, 2018). Other azoles such as voriconazole show variable antifungal activity
(Table 2). While more than $95 \%$ of isolates from India were resistant to a topical allylamine, terbinafine (Chowdhary et al., 2018), currently UK strains remain susceptible to terbinafine and polyene nystatin (Sarma and Upadhyay, 2017). Almost onethird of isolates observed so far were resistant to amphotericin B (a polyene used as a last resort drug) (Warris, 2018). Although 
TABLE 3 | Tentative MIC breakpoints of C. auris defined by CDC (Antifungal Susceptibility Testing and Interpretation, 2019).

\begin{tabular}{lcc}
\hline Drug class & Drug & Tentative MIC breakpoint $(\boldsymbol{\mu} \mathbf{g} / \mathbf{m L})$ \\
\hline Azoles & Fluconazole & $\geq 32$ \\
Azoles & Other azoles & N/A \\
Polyenes & Amphotericin B & $\geq 2$ \\
Echinocandins & Anidulafungin & $\geq 4$ \\
Echinocandins & Caspofungin & $\geq 2$ \\
Echinocandins & Micafungin & $\geq 4$ \\
\hline
\end{tabular}

the nucleoside analog 5-flucytosine successfully treated more than $95 \%$ of $C$. auris infection cases in vitro (Lockhart et al., 2017a; Osei Sekyere, 2018), it is not used extensively as therapy because resistance arises rapidly, potentially during treatment (Rhodes et al., 2018a), and it elicits toxic effects on bone marrow that may lead to death in immunosuppressed patients (Vermes et al., 2000). Between 2 and 7\% of C. auris isolates have developed resistance to echinocandins (Lockhart et al., 2017b; Chowdhary et al., 2018), one the newest classes of antifungal drug to be developed (Sucher et al., 2009). Reported adverse effects of echinocandin therapy are generally mild and include nausea and dose-related elevation of liver aminotransferases levels (Aguilar-Zapata et al., 2015) and therefore, this group of drugs is still the most effective for treating the majority of C. auris infections. Resistance to all three common antifungal classes used as therapy (azoles, polyenes and echinocandins) was observed in $4 \%$ of C. auris outbreak samples in North America from 2012-2015 (Lockhart et al., 2017b).

Although C. auris is typically multidrug-resistant, levels of susceptibility to various drugs differ greatly between isolates and clades (Kean and Ramage, 2019). To find the most suitable drug candidate, susceptibility is typically measured over the period of $24 \mathrm{~h}$, when the fungus grows in the presence of a drug concentration gradient. If the observed minimal inhibitory concentration (MIC) is greater than the resistance MIC breakpoint, the examined isolate is considered resistant to the drug. Tentative susceptibility MIC breakpoints for C. auris established by CDC are shown in Table 3. Currently, these C. auris susceptibility breakpoints should be treated as a general guide as the connection between them and clinical outcome is not known yet.

Importantly, some Candida such as C. albicans exhibit drug tolerance defined as the ability of a fraction of a population to grow above the population resistance level (Rosenberg et al., 2018). The presence of highly tolerant sub-populations may lead to persistent candidemia that is associated with a mortality rate higher than 50\% (Hammoud et al., 2013) despite appropriate treatment (Alatoom et al., 2018). In vivo data suggest that reducing drug susceptibility without affecting the resistance of the population may be possible using adjuvant drugs (Rosenberg et al., 2018). Adjuvants such as the antidepressant fluoxetine that impairs biofilm development (Oliveira et al., 2018) or Hsp90 inhibitor called radicicol that blocks stress responses (Rosenberg et al., 2018) have been shown to clear tolerance without altering the susceptibility level when given in combination with fluconazole in C. albicans (Rosenberg et al., 2018). Future clinical control trials are required to confirm the effectiveness of adjuvants before being considered for inclusion as a possible treatment protocol.

\section{MECHANISMS OF DRUG RESISTANCE IN C. auris}

C. auris has evolved a range of molecular drug-resistance mechanisms which are shown in Figure 1. They include (1) Drug target mutation (Bidaud et al., 2018), (2) drug target overexpression (Bhattacharya et al., 2019), (3) changes in drug uptake and efflux (Muñoz et al., 2018), (4) activation of stress response pathways (Kim et al., 2019), and (5) biofilm formation (Kean et al., 2018). By aggregating into a colony and forming a biofilm, Candida spp. increase their resistance to all currently available antifungals by up to 1000-fold (Taff et al., 2013). Some of the key regulators of fungal biofilm dispersion, antibiotic tolerance, and cell wall remodeling are molecular chaperones belonging to Hsp90 family (Robbins et al., 2011; Leach et al., 2016). In C. auris, these proteins were found to promote cellwall integrity signaling and stress responses related to azoles administration thereby contributing to the evolution of drug resistance (Kim et al., 2019).

Azoles are an important group of antifungal agents that were first developed in the late 1960s, and function by inhibiting the synthesis of ergosterol, a key component of the fungal membrane, thereby preventing growth and proliferation (Ghannoum and Rice, 1999). Since the action of azoles is largely dependent on the affinity of the drug to the active site of Erg11p, any mutations that affect the active site of the enzyme may lead to the development of drug resistance (Berkow and Lockhart, 2017). Such resistance mechanisms have been observed in C. albicans, in which more than 140 ERG11 mutations have been identified to date (Debnath and Addya, 2014). These mutations accumulated in three regions of the ERG11 gene (Marichal et al., 1999). Bioinformatic analysis of these substitutions combined with $3 \mathrm{D}$ modeling showed that most of them are located near the predicted catalytic center or on elements of the fungus-specific external loop (Whaley et al., 2017). Many of the described mutations can also be found in $C$. auris, but not all have the same effects (Muñoz et al., 2018). In both species, amino acid substitutions at F126L, Y132F, K143R result in azole resistance (Morio et al., 2010; Chowdhary et al., 2018; Healey et al., 2018), with $\mathrm{Y} 132 \mathrm{~F}$ the most widespread mutation associated with azole resistance (Chow et al., 2020). Another mechanism of azole resistance is the overexpression of Erg11p, which can be caused either by increasing the number of ERG11 transcription factors such as Upc2p or by duplicating ERG11 (Bhattacharya et al., 2019). In both cases, the high concentration of azole target dilutes the effects of drug activity (Muñoz et al., 2018).

The activity of azoles can be also reduced by decreasing the concentration of antifungals in the cell by means of efflux pumps. Recently, 20 ABC transporters that are putative efflux pumps have been identified in the C. auris genome, strain-type CBS 10913T (Wasi et al., 2019). Two drug transporters (CDR1 and 


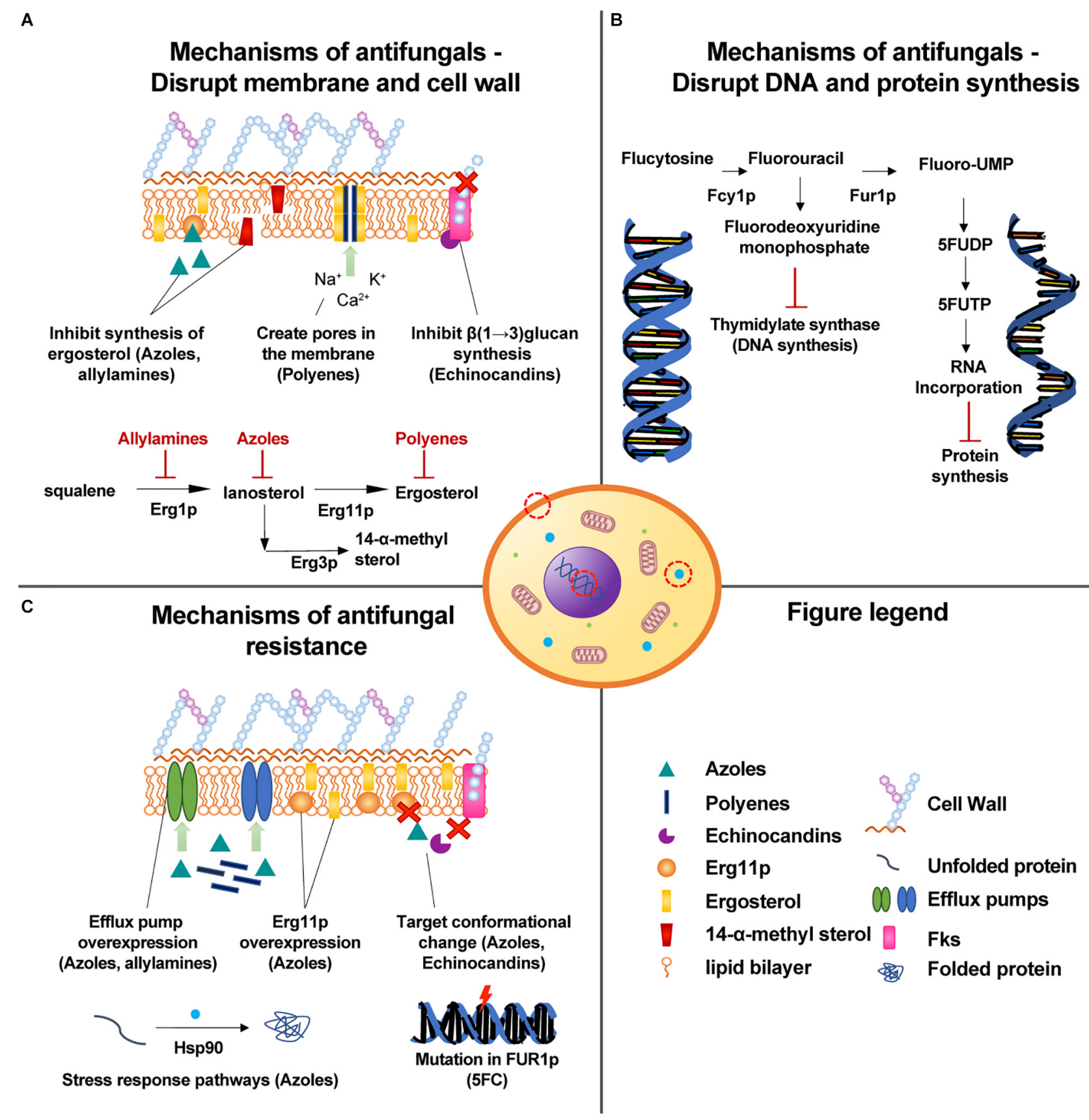

FIGURE 1 | Mechanisms of drug action and resistance observed in C. auris. (A) The main mechanisms of antifungals that disrupt the cell membrane or cell wall. (B) In the nucleus, 5-flucytosine inhibits the synthesis of fungal DNA and RNA. (C) Mechanisms of antifungal resistance to drugs that damage the cell membrane or cell wall.

MDR1) that have primarily been studied in C. albicans have orthologs in C. auris, and these orthologs are overexpressed in azole-resistant C. auris (Rybak et al., 2019). CDR1 is an ABC transporter that confers azole-derived compounds resistance, while MDR1 is a Major Facilitator Superfamily (MFS) pump and a member of the multidrug resistance family which is responsible for fluconazole resistance (de Oliveira Santos et al., 2018). Rybak et al. demonstrated that deletion of CDR1 restored susceptibility of highly resistant C. auris isolates to fluconazole. Furthermore, domains associated with transporters such as OPT and glutathione were found to be significantly enriched in C. auris, C. haemulonii, C. duobushaemulonii, and C. pseudohaemulonii when compared to related species (Muñoz et al., 2018). Oligopeptide transporters (OPT) are small peptide transporters expression of which is upregulated in C. albicans upon administration of azoles (Muñoz et al., 2018). Similarly, 
glutathione transporters may contribute to azole resistance by exporting oxidized glutathione derivatives out of the cell and thereby protecting the cell against oxidative damage elicited by the drugs (Maras et al., 2014).

Polyenes are a commonly used group of drugs used to treat C. auris infections. The most known polyene, amphotericin B, binds membrane-bound ergosterol, causing pores to form in the fungal membrane through which small molecules leak to the outside of the cell (de Oliveira Santos et al., 2018). The release of intracellular constituents such as sugars, potassium or calcium strongly contributes to cell death (de Oliveira Santos et al., 2018). We are not aware of any mechanism of polyenes resistance reported for C. auris so far, which would presumably be analogous to ERG3 dependent alternations in sterol synthesis observed in C. albicans or alternations in cell membrane sterol composition (Taff et al., 2013). However, in 2018, Muñoz et al. (2018) showed that intrinsic transcription of multidrug transporters in C. auris was higher when compared to susceptible and resistant isolates and that upon administering Amphotericin- $B$, eight genes including OPT1-like transporter (high-affinity glutathione, tetra- and pentapeptides transporter), CSA1 (conserved cell wall protein 1 involved in biofilm formation), MET15 (sulfhydrylase) and ARG1 (argininosuccinate synthase) were upregulated. In 2019, another study identified five novel mutations that associate strongly with amphotericin B resistance in Colombian C. auris $(p \leq 0.001)$. Four of the identified mutations fall within proteincoding regions (Escandón et al., 2019). One of the mutations occurs within FLO8, a transcription factor that is required for virulence and biofilm formation in C. albicans (Escandón et al., 2019). Another one is located within a predicted transmembrane protein that could contribute to drug resistance (Escandón et al., 2019). Drug transporters overexpression upon administering amphotericin B was also observed by Wasi et al. In their study, they found 8.7-fold increase in expression of a homolog of CDR6 ABC transporter (Wasi et al., 2019). In the search for more robust drugs and drug targets, we urgently need to better understand the structure and function of drug pumps in pathogenic fungi, as well as how allelic diversity and mutations can affect drug efficacy.

Echinocandins act through the non-competitive inhibition of FKS1 gene product called $\beta(1 \rightarrow 3)$ glucan synthase, which results in stopping production of glucan. Because of depleted glucan, the fungal cell becomes weak and prone to osmotic stress (de Oliveira Santos et al., 2018). C. auris' resistance to echinocandins has been linked to mutations S639F, S639P and S639Y that occur in the highly conserved hot-spot 1 of FKS1 and decrease the sensitivity of the enzyme to the drug (Bidaud et al., 2018; Hou et al., 2019). S639P in FKS1 was recently shown to be the most widespread mutation associated with echinocandin resistance (Chow et al., 2020). A recent multi-omics analysis revealed that although the cell wall remodeling enzymes concentration was lower in C. auris when compared to $C$. albicans, in a drug-resistant $C$. auris isolate (MIC fluconazole: $>256 \mu \mathrm{g} / \mathrm{mL}$, MIC caspofungin: $2 \mu \mathrm{g} / \mathrm{mL}$ ) cell wall integrity proteins were upregulated, suggesting that the cell wall metabolism can be adjusted in response to drug treatment (Zamith-Miranda et al., 2019).
Little is known about C. auris resistance mechanisms to other drug classes such as nucleoside analogs or allylamines (Jeffery-Smith et al., 2018). The nucleoside analogs class contains only 5-flucytosine (5FC) - a drug inhibiting RNA and DNA synthesis in pathogenic fungi (Rapp, 2004). One amino acid substitution (F211I) that is associated with 5FC resistance has been identified in the conserved FUR1 sequence encoding uracil phosphoribosyltransferase (Rhodes et al., 2018b). However, further work is required to understand how such polymorphisms may affect the functioning of the enzyme, which is responsible for the conversion of 5-fluorouracil into 5-fluoro-UMP acid monophosphate. For allylamines, a homolog of $\mathrm{ABC}$ transporter CDR6 was found to be significantly upregulated in drug-resistant C. auris following terbinafine treatment (Wasi et al., 2019).

\section{DETECTION AND NOVEL TREATMENTS OF C. auris}

Successful treatment of $C$. auris infection depends heavily on its accurate identification. In the early stages of infection, symptoms are non-specific and blood cultures typically remain negative (Pfaller and Castanheira, 2016). The resulting delay in diagnosis directly translates to decreased chances of survival; in the study of C. auris outbreak in a European hospital between 2016 and 2017, 41\% of patients died within 1 month from infection (Ruiz-Gaitán et al., 2018).

C. auris does not possess phenotypic features that could easily distinguish it from other Candida spp. (Osei Sekyere, 2018). Depending on the strain it appears purple, red, pink or white on CHROMagar (Kordalewska and Perlin, 2019b) but unlike most types of yeast, it is able to withstand high temperatures $\left(42^{\circ} \mathrm{C}\right)$ (Osei Sekyere, 2018). Many misidentifications related to automated tools such as VITEK 2, BD Phoenix, RapID Yeast Plus, API 20C have been reported to date. Ambaraghassi et al. (2019) demonstrated that VITEK 2 was unable to consistently identify C. auris and that the identification depended on the clade. While the tool identified correctly the South American clade $(n=8)$, its accuracy was variable for isolates from the African $(7 \%, n=10)$ and East Asian clades (0\%, $n=4)$ (Ambaraghassi et al., 2019). Genomics analysis showed that the low identification rate was likely due to the deletion of the L-rhamnose 7-gene cluster in all clades except for the South African clade (Ambaraghassi et al., 2019). As one of 20 tests performed by the VITEK 2 platform is based on the ability of the tested organism to assimilate L-rhamnose (Graf et al., 2000, 2), the deletion of L-rhamnose gene cluster can have an impact on identification results. The most reliable way to identify $C$. auris without genomic sequencing is MALDI-TOF MS (Vatanshenassan et al., 2019). Although not all reference databases contain its protein profile, a free supplemental update is available from MicrobeNet (Lockhart et al., 2017a). A variety of groups have also had success with sub-genomic methods such as sequencing D1-D2 region of 28s ribosomal DNA (rDNA) or internal transcribed spacer region of rDNA (Jeffery-Smith et al., 2018). Unfortunately, access to such tools is limited in many community hospitals. 
TABLE 4 | New antifungals in trials.

\begin{tabular}{|c|c|c|c|c|}
\hline Company & Drug & Trial (Phase) & Activity & References \\
\hline Amplyx & Fosmanogepix (APX001)* & C. auris (Ib), Candidemia (II) & Gwt1 inhibitor (novel) & Hager et al., 2018 \\
\hline Synexis & Ibrexafungerp* & C. auris (III) & $\begin{array}{l}\text { Glucan synthase inhibitor } \\
\text { (novel, orally available) }\end{array}$ & $\begin{array}{l}\text { Berkow and Lockhart, } \\
\text { 2017; Larkin et al., } 2019\end{array}$ \\
\hline NQP 1598 & VT-1598 & C. auris (l) & CYP51 (Erg11p) inhibitor & Wiederhold et al., 2019 \\
\hline Mycovia & $\mathrm{VT}-1161$ & Candidiasis (III) & CYP51 (Erg11p) inhibitor & Brand et al., 2018, p. 2 \\
\hline Cidara & Rezafungin & Candidemia (III) & Long half-life echinocandin & Lepak et al., 2018 \\
\hline
\end{tabular}

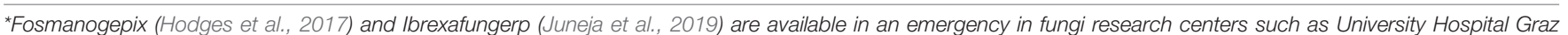
(Graz), University Hospital Innsbruck (Innsbruck), Radboud University (Nijmegen) and University Hospital of Manchester (Manchester).

The necessity for expeditious identification of C. auris in order to effectively treat patients and prevent outbreaks combined with slow current standard laboratory techniques creates a pressing need for new diagnostic tools that could rapidly recognize $C$. auris and its drug resistance patterns. Most of biochemical automated systems and MALDI-TOF MS require cultures, which can take up to 2 weeks to obtain (Kordalewska and Perlin, 2019a). To identify C. auris directly from skin swabs, molecular-based assays such as loop-mediated isothermal amplification (LAMP), T2 magnetic resonance and PCR/qPCR have been applied (Kordalewska and Perlin, 2019b). Since DNA can be isolated directly from the clinical sample, these tests can deliver diagnostic results within several hours (Kordalewska and Perlin, 2019a), which dramatically increases the probability of survival, given that the time window appropriate for initiating appropriate antifungal therapy in Candida bloodstream infection was estimated to $12 \mathrm{~h}$ (Morrell et al., 2005).

The choice of optimal C. auris treatment method depends on both the patient (age, previous use of antifungal medications, infection site, immune status) and the attacking pathogen (strain, possible biofilm formation, drug resistance patterns) (Dodgson et al., 2004; Felton et al., 2014; Bidaud et al., 2018; Moser et al., 2019). The mainstay of therapy is antibiotics. In adults, it is encouraged to begin the therapy with echinocandins even before receiving susceptibility test results (Bidaud et al., 2018). Amphotericin B should be considered for neonates, infants below 2 months of age and as a single or in combination with micafungin when monotherapy with echinocandins fails (Alfouzan et al., 2019; Park et al., 2019). Among azoles, the highest antifungal activity has been observed for itraconazole, isavuconazole and posaconazole (Bidaud et al., 2018), which are sometimes used as a secondary therapy. When voriconazole and micafungin are taken together they show greater effects when compared to the sum of the effects of these drugs taken separately (Cortegiani et al., 2018). While systemic antibiotic treatment is not advised for people colonized with C. auris, control measures should be followed in all cases (Bidaud et al., 2018). Invasive devices such as catheters and ventricular shunts should be removed as soon as they are no longer needed as they may be infected with a biofilm (Bidaud et al., 2018).

C. auris develops resistance rapidly while the patient is being treated and therefore it is crucial to use the available antifungals for the right amount of time and in the right dose (JefferySmith et al., 2018). Close monitoring of drug susceptibility is necessary in both infection and colonization cases in order to identify changes in antifungal resistance (Bidaud et al., 2018). Furthermore, special care is required when administering fungistatic drugs as they may provide the opportunity for resistance to emerge (Berkow and Lockhart, 2017). A small number of new anti-fungal agents are showing successes in clinical trials that may become available for wider use in hospital settings in the upcoming years (Table 4).

\section{MECHANISMS OF VIRULENCE IN C. auris}

Stress resistance, thermotolerance, adherence to host cells, and immune evasion are important virulence traits across Candida species. Investigations of $C$. auris virulence traits often draw comparisons to the extensively-studied pathogen, C. albicans. The $C$. auris genome encodes several orthologs of known virulence factors in C. albicans including genes associated with biofilm formation, antifungal drug resistance, and phenotypic switching (Muñoz et al., 2018). Recent virulence, transcriptome, and other studies are highlighting how these conserved fungal virulence mechanisms may contribute to $C$. auris' emergence as a nosocomial pathogen (Figure 2).

\section{Virulence}

Conflicting reports abound regarding C. auris' virulence in vivo compared to other pathogenic Candida species. Depending on the isolate and infection model used, $C$. auris may be regarded as more virulent (Sherry et al., 2017), as virulent (Borman et al., 2016; Fakhim et al., 2018), or less virulent (BenAmi et al., 2017; Wang et al., 2018) than C. albicans in murine and invertebrate infection models. In studies utilizing the Galleria mellonella invertebrate infection model, C. auris isolates exhibit similar virulence phenotypes, or even slightly more virulent phenotypes, than C. albicans (Borman et al., 2016; Sherry et al., 2017).

Investigators have used immunocompetent and immunocompromised murine models of invasive candidiasis to evaluate C. auris virulence. In immunocompromised animals infected with Israeli $C$. auris clinical isolates, infection lead to rapid death within 5 days, but overall $C$. auris exhibited decreased virulence and lower kidney fungal burdens compared to C. albicans strain CBS 8837 (Ben-Ami et al., 2017). One study with immunocompetent animals established that two clade II C. auris isolates had similar virulence outcomes compared to 


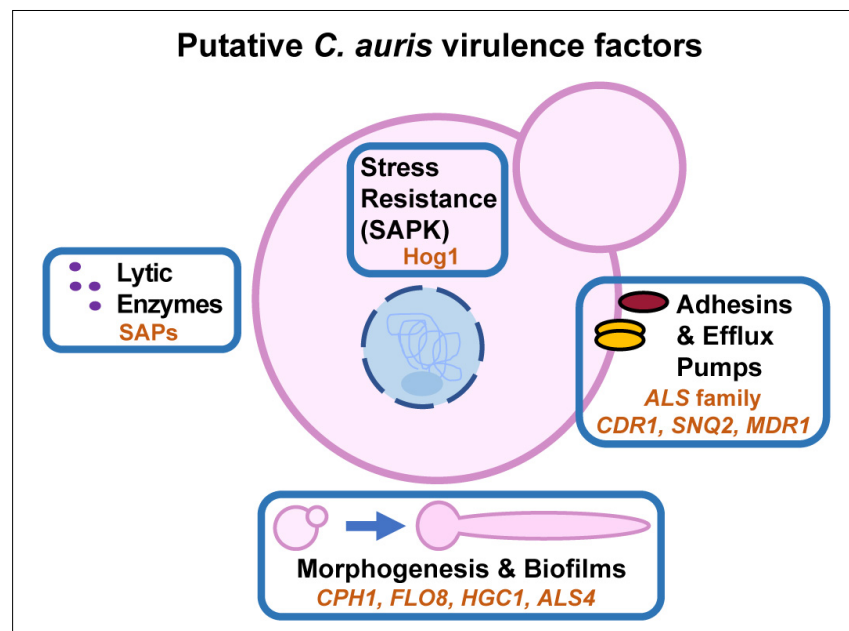

FIGURE 2 | Putative C. auris virulence factors. Key orthologs of C. albicans virulence factors that are transcriptionally induced during $C$. auris growth at $37^{\circ} \mathrm{C}$ are indicated by orange text within each box. C. auris Hog1 is the only conserved ortholog with $C$. albicans that has been experimentally confirmed to play a role in virulence.

one C. albicans clinical isolate (IFRC 1442) (Fakhim et al., 2018), while a study in China determined that the first C. auris isolate identified in China was significantly less virulent than the standard C. albicans laboratory strain SC5314 (Wang et al., 2018). To date, data from murine infection models are difficult to interpret due to inconsistencies in the C. auris and comparator C. albicans strains employed between studies. Consequently, it is not currently possible to speculate whether $C$. auris or individual clades of $C$. auris are generally more, less, or similarly virulent during mammalian infection compared to the prototypic pathogen C. albicans.

Phenotypic and genotypic variability between different C. auris isolates are plausible explanations for the discrepancies described in virulence studies. This variability is somewhat unsurprising given the broad geographical distribution and genetic diversity among clades of $C$. auris outbreaks, but also highlights our poor understanding of $C$. auris pathogenesis. Further characterization of virulence determinants, some of which will be discussed in this section, are important for understanding the virulence potential of this organism. The field would significantly benefit from standardizing virulence investigations by adopting one major C. albicans comparison strain and utilizing high-throughput in vitro methodologies to rapidly identify type strains for each $C$. auris clade that are suitable for comparative in vivo studies.

\section{Lytic Enzymes}

Lytic enzymes, such as secreted aspartyl proteases (SAPs), lipases, phospholipases, and hemolysins, are important virulence factors in human pathogenic fungi (Chaffin, 2008). These enzymes facilitate host cell invasion and tissue destruction by $C$. albicans (Polke et al., 2015). C. auris encodes orthologs of many known C. albicans lytic enzymes, including secreted aspartyl proteases, secreted lipases, and phospholipases (Chatterjee et al., 2015).
Several studies have demonstrated lytic activity for C. auris SAPs (Yue et al., 2018), phospholipases (Larkin et al., 2017), and hemolysin (Kumar et al., 2015), but little is known about how the expression profiles of these enzymes compare to their C. albicans orthologs in vitro or in vivo. Two SAP orthologs were transcriptionally induced in C. auris filamentous cells compared to yeast cells (Yue et al., 2018), but their relative roles in virulence remain unknown. Further molecular and virulence studies are necessary to determine the importance of lytic enzymes to C. auris' infection strategy.

\section{Filamentous Growth}

While filamentation is integral to C. albicans virulence (Zheng et al., 2004), its contribution to C. auris virulence is less certain given that in vivo evidence to date has identified only yeast cells in animal tissues (Ben-Ami et al., 2017). Interestingly, in vivo passage can confer a filamentation-competent (FC) phenotype, but FC and yeast cells have comparable virulence in mice suggesting that filamentation is dispensable for $C$. auris virulence (Yue et al., 2018).

In vitro $C$. auris filamentation occurs under a restricted and/or atypical set of growth conditions compared to C. albicans (Wang et al., 2018; Yue et al., 2018). Like C. albicans, Hsp90 depletion or pharmacological inhibition induces filamentous growth and the expression of adhesins and cell wall genes orthologous to C. albicans filament-associated genes (Kim et al., 2019). During FC-triggered filamentous growth, orthologs of C. albicans CPH1 and FLO8, which encode transcriptional regulators of filamentous growth, were induced in C. auris filamentous cells compared to yeast (Yue et al., 2018). In addition, orthologs of $C$. albicans filament-associated genes were upregulated during $C$. auris filamentation, including the G1 cyclin-related gene HGC1 and glycosylphosphatidylinositol (GPI) anchored gene ALS4. However, there were key differences in the filamentation transcriptomes for $C$. auris relative to C. albicans. EFG1, an important transcriptional regulator of filamentation in C. albicans, was down-regulated in C. auris filamentous cells. Other C. albicans filament-associated cell wall orthologs were preferentially expressed during $C$. auris yeast growth (PGA34, PGA38, and PGA58) (Yue et al., 2018). Thus, while $C$. auris shares some transcriptomic aspects of the C. albicans filamentous growth program, the role of C. auris filamentation and filament-associated genes in its pathogenicity requires further study.

\section{Adherence and Biofilm Formation}

Adherence to host cells is essential to microbial colonization, persistence, and virulence (Mayer et al., 2013; Tsui et al., 2016). In addition, microbe-microbe adherence mediates the formation of microbial communities, or biofilms, which are an important virulence trait in Candida species that confer enhanced antimicrobial resistance. The $C$. auris genome encodes several orthologs of $C$. albicans adhesins implicated in biofilm formation and virulence. Clade II strains of C. auris, which predominantly cause ear infections, have lost significant sections of subtelomeric regions that are enriched in putative adhesins, but are conserved in strains from Clades I, III, and IV (Muñoz et al., 2019). 
Perhaps future studies will unravel what role these subtelomericencoded adhesins have in human colonization and virulence. Recent studies have identified differentially regulated adhesin expression under physiologically-relevant conditions, including growth at $37^{\circ} \mathrm{C}$ in RPMI medium (Kean et al., 2018; Yue et al., 2018).

Several GPI-anchored cell wall proteins contribute to microbe-microbe and microbe-host adherence. ALS4 is a member of the well-characterized ALS adhesin gene family in C. albicans (Zhao et al., 2005) and its ortholog is also differentially expressed during $C$. auris filamentous growth (Yue et al., 2018). Other GPI-anchored cell wall genes and potential adhesins were upregulated during $C$. auris in vitro biofilm formation compared to planktonic cells (IFF4, CSA1, PGA26, PGA52, and HYR3) (Kean et al., 2018). Furthermore, putative orthologs of $A L S 3$, the C. albicans adhesin and invasin that induces host cell endocytosis of C. albicans hyphae (Liu and Filler, 2011), were detected on the C. auris cell surface by an anti-Als3p antibody (Singh et al., 2019). In addition, two ALS family orthologs, ALS1 and ALS5, were expressed in biofilms (Kean et al., 2018). Each adhesin may play an important role in C. auris biofilm formation, persistence in hospital environments, and virulence, but further molecular studies are required to tease apart their independent contributions.

Several efflux pumps were induced in C. auris biofilms versus planktonic cells (Kean et al., 2018). ATP-binding cassette (ABC) transporters $S N Q 2$ and $C D R 1$ and major facilitator superfamily (MFS) genes YHD3, RDC3, and MDR1 were upregulated between 2- and 4 -fold in mature $24 \mathrm{~h}$ biofilms compared to planktonic cells. In line with these observations, efflux pump activity was elevated in mature biofilms relative to planktonic cells (Kean et al., 2018). Altogether, these findings suggest that biofilm formation may promote $C$. auris multi-drug resistance and virulence. Therefore, as with $C$. albicans, clinical measures to tackle $C$. auris drug resistance should consider the possibility that biofilms may harbor more drug-resistant cells and reduce the bioavailability of therapeutics.

\section{Stress Resistance and Persistence}

One of the fascinating traits linked to $C$. auris' emergence as a nosocomial pathogen is its ability to persist on abiotic hospital surfaces under stringent cleaning protocols (Schelenz et al., 2016; Welsh et al., 2017). Viable C. auris can be recovered from plastic surfaces or be detected via esterase activity for up to 2 and 4 weeks post-contamination, respectively (Welsh et al., 2017). These findings pose an interesting question: can C. auris form viable but non-culturable cells that persist in hospital environments? Although no studies to date have directly addressed this question, current metabolomic, transcriptomic, and molecular studies hold clues to $C$. auris' stress tolerance and persistence.

C. auris metabolism favors respiration as evidenced by enrichment in glycolytic and sugar transporter gene expression during yeast growth (Yue et al., 2018) and TCA cycle protein enrichment compared to C. albicans (Zamith-Miranda et al., 2019). Respiratory metabolism enhances ATP production and can reduce oxidative stress in C. albicans, thus promoting in vivo fitness and fluconazole resistance (Guo et al., 2017). Furthermore, C. auris lipid profiles show elevated levels of ergosterol and structural lipids compared to C. albicans, which may influence stress and antifungal resistance (Zamith-Miranda et al., 2019).

In addition, iron transport (Kean et al., 2018) and iron metabolism-associated genes (Yue et al., 2018) were upregulated in C. auris biofilm and filamentous cells, respectively. The C. auris genome provides evidence for expansion of siderophorebased iron transporters compared to $C$. albicans, further highlighting the importance of iron acquisition in this pathogen (Muñoz et al., 2018). Iron is an essential micronutrient that is depleted via nutritional immunity mechanisms in vivo, thus upregulation of iron uptake and metabolism-associated genes may improve C. auris fitness during infection. However, the contribution of $C$. auris iron transport gene families to virulence and drug resistance are currently unknown. Still, the expansion of the iron transport gene family suggests that iron deprivation may play an influential role in C. auris' environmental reservoir.

Recent evidence has demonstrated that the stress-activated protein kinase Hog1 plays an evolutionarily conserved role in C. auris stress resistance, cell wall homeostasis, and virulence (Day et al., 2018). C. auris Hog1 shares 87\% identity with the C. albicans Hog1 sequence. Consistent with findings from other Candida species, C. auris HOG1 was required for osmotic stress resistance and SDS protection. However, C. auris HOG1 was not required for weak acid or nitrosative stress tolerance, which contrasts its role in C. glabrata and C. albicans, respectively (Day et al., 2018). However, we do not know which $C$. auris genes are regulated in a Hog1dependent manner or which Hog1 targets are responsible for these stress-adaptive phenotypes. Future transcriptomics and molecular studies will hopefully reveal which C. auris stress response genes are regulated via Hog1, which will highlight the evolutionary diversification of stress response circuitry across Candida species.

\section{EPIGENETICS AND GENE REGULATION IN C. auris}

Phenotypic plasticity is the interaction between a given genotype and the environment to produce multiple phenotypes. This plasticity is often the result of alterations in gene expression, which can be influenced by genetic or epigenetic mechanisms. In pathogens, phenotypic plasticity is often viewed as system for evolutionary bet-hedging to ensure microbial survival under shifting and/or hostile environmental conditions (Carja and Plotkin, 2017).

In the case of $C$. auris, very little is known about epigenetics and gene regulation or how these contribute to pathogenesis. As with other biological and virulence traits, early insights into $C$. auris epigenetics have been informed by previous work in C. albicans. Chromatin modification, including histone acetylation and deacetylation, plays an important role in C. albicans virulence (Garnaud et al., 2016; Kuchler et al., 2016). C. auris histone proteins and modifying enzymes were 
differentially expressed during filamentous growth relative to yeast, suggesting that epigenetic mechanisms may influence gene expression patterns in yeast and FC cells (Yue et al., 2018). In fact, the observation that in vivo passage can trigger a heritable, filamentation-competent phenotype is suggestive of epigenetic regulation, but more investigation is required to understand the mechanisms underlying this trait and its role in virulence.

Currently, available data demonstrates that $C$. auris has significant similarities in gene expression for important established virulence mechanisms in C. albicans, but there are also significant differences (Kean et al., 2018; Yue et al., 2018). Phenotypic heterogeneity between different C. auris clades provides an opportunity to understand how genetic mechanisms contribute to pathogenesis (via for example gene knock out or mutagenesis studies). Indeed, the adaptation of C. albicans gene deletion technologies for use in C. auris will enable future molecular investigations to understand gene regulatory networks and virulence in this emerging pathogen. Consequently, the C. albicans NAT1-Clox system has been successfully employed to disrupt C. auris HOG1 and demonstrate its conserved role in stress responses (Shahana et al., 2014; Day et al., 2018). Future pathfinding molecular studies should reveal the conservation and divergence of gene regulatory networks in $C$. albicans and C. auris and highlight new avenues for antifungal development.

\section{REFERENCES}

Abdalhamid, B., Almaghrabi, R., Althawadi, S., and Omrani, A. (2018). First report of Candida auris infections from Saudi Arabia. J. Infect. Public Health 11, 598-599. doi: 10.1016/j.jiph.2018.05.010

Abram, N. J., McGregor, H. V., Tierney, J. E., Evans, M. N., McKay, N. P., Kaufman, D. S., et al. (2016). Early onset of industrial-era warming across the oceans and continents. Nature 536, 411-418. doi: 10.1038/nature19082

Adam, R. D., Revathi, G., Okinda, N., Fontaine, M., Shah, J., Kagotho, E., et al. (2019). Analysis of Candida auris fungemia at a single facility in Kenya. Int. J. Infect. Dis. IJID Off. Publ. Int. Soc. Infect. Dis. 85, 182-187. doi: 10.1016/j.ijid. 2019.06.001

Aguilar-Zapata, D., Petraitiene, R., and Petraitis, V. (2015). Echinocandins: the expanding antifungal armamentarium. Clin. Infect. Dis. Off. Publ. Infect. Dis. Soc. Am. 61(Suppl. 6), S604-S611. doi: 10.1093/cid/civ814

Alatoom, A., Sartawi, M., Lawlor, K., AbdelWareth, L., Thomsen, J., Nusair, A., et al. (2018). Persistent candidemia despite appropriate fungal therapy: first case of Candida auris from the United Arab Emirates. Int. J. Infect. Dis. IJID Off. Publ. Int. Soc. Infect. Dis. 70, 36-37. doi: 10.1016/j.ijid.2018.02.005

Alfouzan, W., Dhar, R., Albarrag, A., and Al-Abdely, H. (2019). The emerging pathogen Candida auris: a focus on the middle-Eastern countries. J. Infect. Public Health 12, 451-459. doi: 10.1016/j.jiph.2019.03.009

Al-Yasiri, M. H., Normand, A.-C., L'Ollivier, C., Lachaud, L., Bourgeois, N., Rebaudet, S., et al. (2016). Opportunistic fungal pathogen Candida glabrata circulates between humans and yellow-legged gulls. Sci. Rep. 6:36157. doi: 10 . 1038/srep36157

Ambaraghassi, G., Dufresne, P. J., Dufresne, S. F., Vallières, É, Muñoz, J. F., Cuomo, C. A., et al. (2019). Identification of Candida auris by use of the updated Vitek 2 yeast identification system, version 8.01: a multilaboratory evaluation study. J. Clin. Microbiol. 57:e00884-19. doi: 10.1128/JCM.00884-19

Angoulvant, A., Guitard, J., and Hennequin, C. (2016). Old and new pathogenic Nakaseomyces species: epidemiology, biology, identification, pathogenicity and antifungal resistance. FEMS Yeast Res. 16:fov114. doi: 10.1093/femsyr/ fov114

\section{SUMMARY}

Genomics and other omics studies have provided significant insight into the biology and pathogenesis of $C$. auris since its emergence as a modern human fungal pathogen. However, several questions remain about the evolution and fitness of this multi-drug resistant yeast which require further study.

\section{AUTHOR CONTRIBUTIONS}

All authors wrote and reviewed the manuscript and contributed and worked on figures.

\section{FUNDING}

RF was supported by MRC grant MR/N006364/2 and a Wellcome Trust Seed Award (215239/Z/19/Z).

\section{ACKNOWLEDGMENTS}

We would like to give thanks to Carolina Coelho for helpful discussions and assistance with Figure 1 and Jose Muñoz for valuable feedback on the manuscript.

Antifungal Susceptibility Testing and Interpretation (2019). Available online at: https://www.cdc.gov/fungal/candida-auris/c-auris-antifungal.html (accessed November 26, 2019). doi: 10.1093/femsyr/fov114

Araúz, A. B., Caceres, D. H., Santiago, E., Armstrong, P., Arosemena, S., Ramos, C., et al. (2018). Isolation of Candida auris from 9 patients in Central America: importance of accurate diagnosis and susceptibility testing. Mycoses 61, 44-47. doi: 10.1111/myc.12709

Arendrup, M. C., Prakash, A., Meletiadis, J., Sharma, C., and Chowdhary, A. (2017). Comparison of EUCAST and CLSI reference microdilution MICs of eight antifungal compounds for Candida auris and associated tentative epidemiological cutoff values. Antimicrob. Agents Chemother. 61:e00485-17. doi: 10.1128/AAC.00485-17

Aulie, A. (1971). Body temperatures in pigeons and budgerigars during sustained flight. Comp. Biochem. Physiol. A Physiol. 39, 173-176. doi: 10.1016/03009629(71)90074-0

Bandeira, A. C., Filho, J. M., and de Almeida Ramos, K. (2016). Reversible cardiomyopathy secondary to Amphotericin-B. Med. Mycol. Case Rep. 13, 19-21. doi: 10.1016/j.mmcr.2016.10.001

Barantsevich, N. E., Orlova, O. E., Shlyakhto, E. V., Johnson, E. M., Woodford, N., Lass-Floerl, C., et al. (2019). Emergence of Candida auris in Russia. J. Hosp. Infect. 102, 445-448. doi: 10.1016/j.jhin.2019.02.021

Ben-Ami, R., Berman, J., Novikov, A., Bash, E., Shachor-Meyouhas, Y., Zakin, S., et al. (2017). Multidrug-resistant Candida haemulonii and C. auris, Tel Aviv, Israel. Emerg. Infect. Dis. 23, 195-203. doi: 10.3201/eid2302.161486

Berkow, E. L., and Lockhart, S. R. (2017). Fluconazole resistance in Candida species: a current perspective. Infect. Drug Resist. 10, 237-245. doi: 10.2147/IDR. S118892

Bhattacharya, S., Holowka, T., Orner, E. P., and Fries, B. C. (2019). Gene duplication associated with increased Fluconazole tolerance in Candida auris cells of advanced generational age. Sci. Rep. 9:5052. doi: 10.1038/s41598-01941513-6

Bidaud, A. L., Chowdhary, A., and Dannaoui, E. (2018). Candida auris: an emerging drug resistant yeast - a mini-review. J. Mycol. Medicale 28, 568-573. doi: 10.1016/j.mycmed.2018.06.007 
Borman, A. M., Szekely, A., and Johnson, E. M. (2016). Comparative pathogenicity of United Kingdom isolates of the emerging pathogen Candida auris and other key pathogenic Candida species. $m$ Sphere 1:e00189-16. doi: 10.1128/mSphere. 00189-16

Brand, S. R., Degenhardt, T. P., Person, K., Sobel, J. D., Nyirjesy, P., Schotzinger, R. J., et al. (2018). A phase 2, randomized, double-blind, placebo-controlled, dose-ranging study to evaluate the efficacy and safety of orally administered VT-1161 in the treatment of recurrent vulvovaginal candidiasis. Am. J. Obstet. Gynecol. 218, 624.e1-624.e9. doi: 10.1016/j.ajog.2018.03.001

Byrnes, E. J. III, Li, W., Lewit, Y., Ma, H., Voelz, K., Ren, P., et al. (2010). Emergence and pathogenicity of highly virulent Cryptococcus gattii genotypes in the Northwest United States. PLoS Pathog. 6:e1000850. doi: 10.1371/journal. ppat. 1000850

Carja, O., and Plotkin, J. B. (2017). The evolutionary advantage of heritable phenotypic heterogeneity. Sci. Rep. 7:5090. doi: 10.1038/s41598-017-05214-2

Casadevall, A., Kontoyiannis, D. P., and Robert, V. (2019). On the emergence of Candida auris: climate change, azoles, swamps, and birds. mBio 10:e01397-19. doi: 10.1128/mBio.01397-19

Cendejas-Bueno, E., Kolecka, A., Alastruey-Izquierdo, A., Theelen, B., Groenewald, M., Kostrzewa, M., et al. (2012). Reclassification of the Candida haemulonii complex as Candida haemulonii (C. haemulonii Group I), C. duobushaemulonii sp. nov. (C. haemulonii Group II), and C. haemulonii var. vulnera var. nov.: three multiresistant human pathogenic yeasts. J. Clin. Microbiol. 50, 3641-3651. doi: 10.1128/JCM.02248-12

Chaffin, W. L. (2008). Candida albicans cell wall proteins. Microbiol. Mol. Biol. Rev. MMBR 72, 495-544. doi: 10.1128/MMBR.00032-07

Chatterjee, S., Alampalli, S. V., Nageshan, R. K., Chettiar, S. T., Joshi, S., and Tatu, U. S. (2015). Draft genome of a commonly misdiagnosed multidrug resistant pathogen Candida auris. BMC Genomics 16:686. doi: 10.1186/s12864015-1863-z

Chow, N. A., de Groot, T., Badali, H., Abastabar, M., Chiller, T. M., and Meis, J. F. (2019). Potential fifth clade of Candida auris. Emerg. Infect. Dis. 25, 1780-1781. doi: 10.3201/eid2509.190686

Chow, N. A., Gade, L., Tsay, S. V., Forsberg, K., Greenko, J. A., Southwick, K. L., et al. (2018). Multiple introductions and subsequent transmission of multidrugresistant Candida auris in the USA: a molecular epidemiological survey. Lancet Infect. Dis. 18, 1377-1384. doi: 10.1016/S1473-3099(18)30597-8

Chow, N. A., Muñoz, J. F., Gade, L., Berkow, E., Li, X., Welsh, R. M., et al. (2020). Tracing the evolutionary history and global expansion of Candida auris using population genomic analyses. bioRxiv [Preprint]. doi: 10.1101/2020.01. 06.896548

Chowdhary, A., Prakash, A., Sharma, C., Kordalewska, M., Kumar, A., Sarma, S., et al. (2018). A multicentre study of antifungal susceptibility patterns among 350 Candida auris isolates (2009-17) in India: role of the ERG11 and FKS1 genes in azole and echinocandin resistance. J. Antimicrob. Chemother. 73, 891-899. doi: $10.1093 / \mathrm{jac} / \mathrm{dkx} 480$

Cortegiani, A., Misseri, G., Fasciana, T., Giammanco, A., Giarratano, A., and Chowdhary, A. (2018). Epidemiology, clinical characteristics, resistance, and treatment of infections by Candida auris. J. Intensive Care 6:69. doi: 10.1186/ s40560-018-0342-4

Day, A. M., McNiff, M. M., da Silva Dantas, A., Gow, N. A. R., and Quinn, J. (2018). Hog1 regulates stress tolerance and virulence in the emerging fungal pathogen Candida auris. $m$ Sphere 3:e0506-18. doi: 10.1128/mSphere.00506-18

de Oliveira Santos, G. C., Vasconcelos, C. C., Lopes, A. J. O., de Sousa Cartágenes, M., do, S., Filho, A. K. D. B., et al. (2018). Candida infections and therapeutic strategies: mechanisms of action for traditional and alternative agents. Front. Microbiol. 9:1351. doi: 10.3389/fmicb.2018.01351

Debnath, S., and Addya, S. (2014). Structural basis for heterogeneous phenotype of ERG11 dependent Azole resistance in C. albicans clinical isolates. SpringerPlus 3:660. doi: 10.1186/2193-1801-3-660

Dodgson, A. R., Dodgson, K. J., Pujol, C., Pfaller, M. A., and Soll, D. R. (2004). Clade-specific flucytosine resistance is due to a single nucleotide change in the FUR1 gene of Candida albicans. Antimicrob. Agents Chemother. 48, 2223-2227. doi: 10.1128/AAC.48.6.2223-2227.2004

Dukik, K., Muñoz, J. F., Jiang, Y., Feng, P., Sigler, L., Stielow, J. B., et al. (2017). Novel taxa of thermally dimorphic systemic pathogens in the Ajellomycetaceae (Onygenales). Mycoses 60, 296-309. doi: 10.1111/myc. 12601
Escandón, P., Chow, N. A., Caceres, D. H., Gade, L., Berkow, E. L., Armstrong, P., et al. (2019). Molecular epidemiology of Candida auris in Colombia reveals a highly related, countrywide colonization with regional patterns in Amphotericin B resistance. Clin. Infect. Dis. Off. Publ. Infect. Dis. Soc. Am. 68, 15-21. doi: 10.1093/cid/ciy411

Fakhim, H., Vaezi, A., Dannaoui, E., Chowdhary, A., Nasiry, D., Faeli, L., et al. (2018). Comparative virulence of Candida auris with Candida haemulonii, Candida glabrata and Candida albicans in a murine model. Mycoses 61, 377382. doi: $10.1111 /$ myc. 12754

Farrer, R. A. (2019). Batrachochytrium salamandrivorans. Trends Microbiol. 27, 892-893. doi: 10.1016/j.tim.2019.04.009

Farrer, R. A., and Fisher, M. C. (2017). Describing genomic and epigenomic traits underpinning emerging fungal pathogens. Adv. Genet. 100, 73-140. doi: 10. 1016/bs.adgen.2017.09.009

Felton, T., Troke, P. F., and Hope, W. W. (2014). Tissue penetration of antifungal agents. Clin. Microbiol. Rev. 27, 68-88. doi: 10.1128/CMR.00046-13

Fraser, J. A., Giles, S. S., Wenink, E. C., Geunes-Boyer, S. G., Wright, J. R., Diezmann, S., et al. (2005). Same-sex mating and the origin of the Vancouver Island Cryptococcus gattii outbreak. Nature 437, 1360-1364. doi: 10.1038/ nature 04220

Garcia-Solache, M. A., and Casadevall, A. (2010). Global warming will bring new fungal diseases for mammals. mBio 1:e00061-10. doi: 10.1128/mBio.00061-10

Garnaud, C., Champleboux, M., Maubon, D., Cornet, M., and Govin, J. (2016). Histone deacetylases and their inhibition in Candida species. Front. Microbiol. 7:1238. doi: 10.3389/fmicb.2016.01238

Ghannoum, M. A., and Rice, L. B. (1999). Antifungal agents: mode of action, mechanisms of resistance, and correlation of these mechanisms with bacterial resistance. Clin. Microbiol. Rev. 12, 501-517. doi: 10.1128/cmr.12.4.501

Gleeson, M. (1998). Temperature regulation during exercise. Int. J. Sports Med. 19(Suppl. 2), S96-S99. doi: 10.1055/s-2007-971967

Govender, N. P., Magobo, R. E., Mpembe, R., Mhlanga, M., Matlapeng, P., Corcoran, C., et al. (2018). Candida auris in South Africa, 2012-2016. Emerg. Infect. Dis. 24, 2036-2040. doi: 10.3201/eid2411.180368

Graf, B., Adam, T., Zill, E., and Göbel, U. B. (2000). Evaluation of the VITEK 2 system for rapid identification of yeasts and yeast-like organisms. J. Clin. Microbiol. 38, 1782-1785. doi: 10.1128/jcm.38.5.1782-1785.2000

Guo, H., Xie, S., Li, S., Song, Y., Zhong, X., and Zhang, H. (2017). Involvement of mitochondrial aerobic respiratory activity in efflux-mediated resistance of C. albicans to fluconazole. J. Mycol. Med. 27, 339-344. doi: 10.1016/j.mycmed. 2017.04.004

Hager, C. L., Larkin, E. L., Long, L., Zohra Abidi, F., Shaw, K. J., and Ghannoum, M. A. (2018). In Vitro and In Vivo evaluation of the antifungal activity of APX001A/APX001 against Candida auris. Antimicrob. Agents Chemother. 62:e02319-17. doi: 10.1128/AAC.02319-17

Hammoud, M. S., Al-Taiar, A., Fouad, M., Raina, A., and Khan, Z. (2013). Persistent candidemia in neonatal care units: risk factors and clinical significance. Int. J. Infect. Dis. IJID Off. Publ. Int. Soc. Infect. Dis. 17, e624-e628. doi: 10.1016/j.ijid. 2012.11.020

Hamprecht, A., Barber, A. E., Mellinghoff, S. C., Thelen, P., Walther, G., Yu, Y., et al. (2019). Candida auris in Germany and previous exposure to foreign healthcare. Emerg. Infect. Dis. 25, 1763-1765. doi: 10.3201/eid2509.19 0262

Healey, K. R., Kordalewska, M., Jiménez Ortigosa, C., Singh, A., Berrío, I., Chowdhary, A., et al. (2018). Limited ERG11 mutations identified in isolates of Candida auris directly contribute to reduced azole susceptibility. Antimicrob. Agents Chemother. 62:e1427-18. doi: 10.1128/AAC.01427-18

Heath, C. H., Dyer, J. R., Pang, S., Coombs, G. W., and Gardam, D. J. (2019). Candida auris sternal osteomyelitis in a man from Kenya visiting Australia, 2015. Emerg. Infect. Dis. 25, 192-194. doi: 10.3201/eid2501.181321

Hodges, M. R., Ople, E., Shaw, K. J., Mansbach, R., Van Marle, S. J., Van Hoogdalem, E.-J., et al. (2017). First-in-human study to assess safety, tolerability and pharmacokinetics of APX001 administered by intravenous infusion to healthy subjects. Open Forum Infect. Dis. 4, S526-S526. doi: 10.1093/ofid/ of 163.1370

Hou, X., Lee, A., Jiménez-Ortigosa, C., Kordalewska, M., Perlin, D. S., and Zhao, Y. (2019). Rapid detection of ERG11-associated azole resistance and FKS-associated echinocandin resistance in Candida auris. Antimicrob. Agents Chemother. 63:e1811-18. doi: 10.1128/AAC.01811-18 
Jackson, B. R., Chow, N., Forsberg, K., Litvintseva, A. P., Lockhart, S. R., Welsh, R., et al. (2019). On the origins of a species: what might explain the rise of Candida auris? J. Fungi Basel Switz 5:58. doi: 10.3390/jof5030058

Jeffery-Smith, A., Taori, S. K., Schelenz, S., Jeffery, K., Johnson, E. M., Borman, A., et al. (2018). Candida auris: a review of the Literature. Clin. Microbiol. Rev. 31:e00029-17. doi: 10.1128/CMR.00029-17

Juneja, D., Singh, O., Tarai, B., and Angulo, D. A. (2019). "Successful treatment of two patients with Candida auris candidemia with the investigational agent, oral Ibrexafungerp (formerly SCY-078), from the CARES Study," in Poster at the 29th European Congress of Clinical Microbiology and Infectious Diseases with Amsterdam, The Netherlands.

Kamiñski, D. M. (2014). Recent progress in the study of the interactions of amphotericin B with cholesterol and ergosterol in lipid environments. Eur. Biophys. J. EBJ 43, 453-467. doi: 10.1007/s00249-014-0983-8

Kathuria, S., Singh, P. K., Sharma, C., Prakash, A., Masih, A., Kumar, A., et al. (2015). Multidrug-resistant Candida auris misidentified as Candida haemulonii: characterization by matrix-assisted laser desorption ionizationtime of flight mass spectrometry and DNA sequencing and Its antifungal susceptibility profile variability by Vitek 2, CLSI broth microdilution, and etest method. J. Clin. Microbiol. 53, 1823-1830. doi: 10.1128/JCM.00367-15

Kean, R., Delaney, C., Sherry, L., Borman, A., Johnson, E. M., Richardson, M. D., et al. (2018). Transcriptome assembly and profiling of Candida auris reveals novel insights into biofilm-mediated resistance. mSphere 3:e00334-18. doi: 10. 1128/mSphere.00334- 18

Kean, R., and Ramage, G. (2019). Combined antifungal resistance and biofilm tolerance: the global threat of Candida auris. mSphere 4:e0458-19. doi: 10. 1128/mSphere.00458-19

Khan, Z., Ahmad, S., Benwan, K., Purohit, P., Al-Obaid, I., Bafna, R., et al. (2018). Invasive Candida auris infections in Kuwait hospitals: epidemiology, antifungal treatment and outcome. Infection 46, 641-650. doi: 10.1007/s15010-018$1164-y$

Khan, Z. U., Al-Sweih, N. A., Ahmad, S., Al-Kazemi, N., Khan, S., Joseph, L., et al. (2007). Outbreak of fungemia among neonates caused by Candida haemulonii resistant to amphotericin B, itraconazole, and fluconazole. J. Clin. Microbiol. 45, 2025-2027. doi: 10.1128/JCM.00222-07

Kim, S. H., Iyer, K. R., Pardeshi, L., Muñoz, J. F., Robbins, N., Cuomo, C. A., et al. (2019). Genetic analysis of Candida auris implicates Hsp90 in morphogenesis and azole tolerance and Cdr1 in azole resistance. mBio 10:e02529-18. doi: 10 . 1128/mBio.02529-18

Kohlenberg, A., Struelens, M. J., Monnet, D. L., and Plachouras, D. (2018). Candida auris: epidemiological situation, laboratory capacity and preparedness in European Union and European Economic Area countries, 2013 to 2017. Eurosurveillance 23:18-00136. doi: 10.2807/1560-7917.ES.2018.23.13.18-00136

Kordalewska, M., and Perlin, D. S. (2019a). Identification of drug resistant Candida auris. Front. Microbiol. 10:1918. doi: 10.3389/fmicb.2019.01918

Kordalewska, M., and Perlin, D. S. (2019b). Molecular diagnostics in the times of surveillance for Candida auris. J. Fungi 5:77. doi: 10.3390/jof5030077

Kuchler, K., Jenull, S., Shivarathri, R., and Chauhan, N. (2016). Fungal KATs/KDACs: a new highway to better antifungal drugs? PLoS Pathog. 12:e1005938. doi: 10.1371/journal.ppat.1005938

Kumar, D., Banerjee, T., Pratap, C. B., and Tilak, R. (2015). Itraconazole-resistant Candida auris with phospholipase, proteinase and hemolysin activity from a case of vulvovaginitis. J. Infect. Dev. Ctries. 9, 435-437. doi: 10.3855/jidc.4582

Kwon, Y. J., Shin, J. H., Byun, S. A., Choi, M. J., Won, E. J., Lee, D., et al. (2019). Candida auris clinical isolates from South Korea: identification, antifungal susceptibility, and genotyping. J. Clin. Microbiol. 57:e01624-18. doi: 10.1128/ JCM.01624-18

Larkin, E., Hager, C., Chandra, J., Mukherjee, P. K., Retuerto, M., Salem, I., et al. (2017). The emerging pathogen Candida auris: growth phenotype, virulence factors, activity of antifungals, and effect of SCY-078, a novel glucan synthesis inhibitor, on growth morphology and biofilm formation. Antimicrob. Agents Chemother. 61:e02396-16. doi: 10.1128/AAC.02396-16

Larkin, E. L., Long, L., Isham, N., Borroto-Esoda, K., Barat, S., Angulo, D., et al. (2019). A novel 1,3-Beta-d-Glucan Inhibitor, Ibrexafungerp (Formerly SCY078), shows potent activity in the lower $\mathrm{pH}$ environment of vulvovaginitis. Antimicrob. Agents Chemother. 63:e02611-18. doi: 10.1128/AAC.02611-18

Leach, M. D., Farrer, R. A., Tan, K., Miao, Z., Walker, L. A., Cuomo, C. A., et al. (2016). Hsf1 and Hsp90 orchestrate temperature-dependent global transcriptional remodelling and chromatin architecture in Candida albicans. Nat. Commun. 7:11704. doi: 10.1038/ncomms11704

Lee, W. G., Shin, J. H., Uh, Y., Kang, M. G., Kim, S. H., Park, K. H., et al. (2011). First three reported cases of nosocomial fungemia caused by Candida auris. J. Clin. Microbiol. 49, 3139-3142. doi: 10.1128/JCM.00319-11

Lehmann, P. F., Wu, L. C., Pruitt, W. R., Meyer, S. A., and Ahearn, D. G. (1993). Unrelatedness of groups of yeasts within the Candida haemulonii complex. J. Clin. Microbiol. 31, 1683-1687. doi: 10.1128/jcm.31.7.1683-1687.1993

Lepak, A. J., Zhao, M., and Andes, D. R. (2018). Pharmacodynamic evaluation of Rezafungin (CD101) against Candida auris in the neutropenic mouse invasive candidiasis model. Antimicrob. Agents Chemother. 62:e01572-18. doi: 10.1128/ AAC.01572-18

Liu, Y., and Filler, S. G. (2011). Candida albicans Als3, a multifunctional adhesin and invasin. Eukaryot. Cell 10, 168-173. doi: 10.1128/EC.00279-10

Lockhart, S. R., Berkow, E. L., Chow, N., and Welsh, R. M. (2017a). Candida auris for the clinical microbiology laboratory: not your grandfather's Candida species. Clin. Microbiol. Newsl. 39, 99-103. doi: 10.1016/j.clinmicnews.2017.06.003

Lockhart, S. R., Etienne, K. A., Vallabhaneni, S., Farooqi, J., Chowdhary, A., Govender, N. P., et al. (2017b). Simultaneous emergence of multidrug-resistant Candida auris on 3 continents Cconfirmed by whole-genome sequencing and epidemiological analyses. Clin. Infect. Dis. Off. Publ. Infect. Dis. Soc. Am. 64, 134-140. doi: 10.1093/cid/ciw691

Maras, B., Angiolella, L., Mignogna, G., Vavala, E., Macone, A., Colone, M., et al. (2014). Glutathione metabolism in Candida albicans resistant strains to fluconazole and micafungin. PLoS One 9:e98387. doi: 10.1371/journal.pone. 0098387

Marichal, P., Koymans, L., Willemsens, S., Bellens, D., Verhasselt, P., Luyten, W., et al. (1999). Contribution of mutations in the cytochrome P450 14alphademethylase (Erg11p, Cyp51p) to azole resistance in Candida albicans. Microbiol. Read. Engl. 145(Pt 10), 2701-2713. doi: 10.1099/00221287-145-102701

Mayer, F. L., Wilson, D., and Hube, B. (2013). Candida albicans pathogenicity mechanisms. Virulence 4, 119-128. doi: 10.4161/viru.22913

Mohd Tap, R., Lim, T. C., Kamarudin, N. A., Ginsapu, S. J., Abd Razak, M. F., Ahmad, N., et al. (2018). A fatal case of Candida auris and Candida tropicalis candidemia in neutropenic patient. Mycopathologia 183, 559-564. doi: 10.1007/ s11046-018-0244-y

Mohsin, J., Hagen, F., Al-Balushi, Z. A. M., de Hoog, G. S., Chowdhary, A., Meis, J. F., et al. (2017). The first cases of Candida auris candidaemia in Oman. Mycoses 60, 569-575. doi: 10.1111/myc.12647

Morio, F., Loge, C., Besse, B., Hennequin, C., and Le Pape, P. (2010). Screening for amino acid substitutions in the Candida albicans Erg11 protein of azolesusceptible and azole-resistant clinical isolates: new substitutions and a review of the literature. Diagn. Microbiol. Infect. Dis. 66, 373-384. doi: 10.1016/j. diagmicrobio.2009.11.006

Morrell, M., Fraser, V. J., and Kollef, M. H. (2005). Delaying the empiric treatment of Candida bloodstream infection until positive blood culture results are obtained: a potential risk factor for hospital mortality. Antimicrob. Agents Chemother. 49, 3640-3645. doi: 10.1128/AAC.49.9.3640-3645.2005

Morse, S. S. (1995). Factors in the emergence of infectious diseases. Emerg. Infect. Dis. 1, 7-15. doi: 10.3201/eid0101.950102

Moser, C., Lerche, C. J., Thomsen, K., Hartvig, T., Schierbeck, J., Jensen, P. Ø, et al. (2019). Antibiotic therapy as personalized medicine - general considerations and complicating factors. APMIS 127, 361-371. doi: 10.1111/apm. 12951

Muñoz, J. F., Gade, L., Chow, N. A., Loparev, V. N., Juieng, P., Berkow, E. L., et al. (2018). Genomic insights into multidrug-resistance, mating and virulence in Candida auris and related emerging species. Nat. Commun. 9:5346. doi: 10.1038/s41467-018-07779-6

Muñoz, J. F., Welsh, R. M., Shea, T., Batra, D., Gade, L., Litvintseva, A. P., et al. (2019). Chromosomal rearrangements and loss of subtelomeric adhesins linked to clade-specific phenotypes in Candida auris. bioRxiv [Preprint]. doi: 10.1101/ 754143

Oliveira, A. S., Martinez-de-Oliveira, J., Donders, G. G. G., Palmeira-deOliveira, R., and Palmeira-de-Oliveira, A. (2018). Anti-Candida activity of antidepressants sertraline and fluoxetine: effect upon pre-formed biofilms. Med. Microbiol. Immunol. (Berl.) 207, 195-200. doi: 10.1007/s00430-0180539-0 
Ong, C. W., Chen, S. C.-A., Clark, J. E., Halliday, C. L., Kidd, S. E., Marriott, D. J., et al. (2019). Diagnosis, management and prevention of Candida auris in hospitals: position statement of the Australasian Society for Infectious Diseases. Intern. Med. J. 49, 1229-1243. doi: 10.1111/imj.14612

Osei Sekyere, J. (2018). Candida auris: a systematic review and metaanalysis of current updates on an emerging multidrug-resistant pathogen. MicrobiologyOpen 7:e00578. doi: 10.1002/mbo3.578

Park, J. Y., Bradley, N., Brooks, S., Burney, S., and Wassner, C. (2019). Management of patients with Candida auris fungemia at community hospital, Brooklyn, New York, USA, 2016-2018. Emerg. Infect. Dis. 25, 601-602. doi: 10.3201/ eid2503.180927

Pfaller, M. A., and Castanheira, M. (2016). Nosocomial candidiasis: antifungal stewardship and the importance of rapid diagnosis. Med. Mycol. 54, 1-22. doi: $10.1093 / \mathrm{mmy} / \mathrm{myv076}$

Pfaller, M. A., and Diekema, D. J. (2007). Epidemiology of Invasive candidiasis: a persistent public health problem. Clin. Microbiol. Rev. 20, 133-163. doi: 10. 1128/CMR.00029-06

Pfaller, M. A., Diekema, D. J., Turnidge, J. D., Castanheira, M., and Jones, R. N. (2019). Twenty years of the SENTRY antifungal surveillance program: results for Candida species from 1997-2016. Open Forum Infect. Dis. 6, S79-S94. doi: 10.1093/ofid/ofy358

Polke, M., Hube, B., and Jacobsen, I. D. (2015). Candida survival strategies. Adv. Appl. Microbiol. 91, 139-235. doi: 10.1016/bs.aambs.2014.12.002

Rapp, R. P. (2004). Changing strategies for the management of invasive fungal infections. Pharmacotherapy 24, 4S-28S. doi: 10.1592/phco.24.3.4s.33151

Rhodes, J., Abdolrasouli, A., Farrer, R. A., Cuomo, C. A., Aanensen, D. M., Armstrong-James, D., et al. (2018a). Author correction: genomic epidemiology of the UK outbreak of the emerging human fungal pathogen Candida auris. Emerg. Microbes Infect. 7:104. doi: 10.1038/s41426-018-0098-x

Rhodes, J., Abdolrasouli, A., Farrer, R. A., Cuomo, C. A., Aanensen, D. M., Armstrong-James, D., et al. (2018b). Genomic epidemiology of the UK outbreak of the emerging human fungal pathogen Candida auris. Emerg. Microbes Infect 7:43. doi: 10.1038/s41426-018-0045-x

Riat, A., Neofytos, D., Coste, A., Harbarth, S., Bizzini, A., Grandbastien, B., et al. (2018). First case of Candida auris in Switzerland: discussion about preventive strategies. Swiss Med. Wkly. 148:w14622. doi: 10.4414/smw.2018.14622

Robbins, N., Uppuluri, P., Nett, J., Rajendran, R., Ramage, G., Lopez-Ribot, J. L., et al. (2011). Hsp90 governs dispersion and drug resistance of fungal biofilms. PLoS Pathog. 7:e1002257. doi: 10.1371/journal.ppat.1002257

Rosenberg, A., Ene, I. V., Bibi, M., Zakin, S., Segal, E. S., Ziv, N., et al. (2018). Antifungal tolerance is a subpopulation effect distinct from resistance and is associated with persistent candidemia. Nat. Commun. 9:2470. doi: 10.1038/ s41467-018-04926-x

Ruiz-Gaitán, A., Moret, A. M., Tasias-Pitarch, M., Aleixandre-López, A. I., Martínez-Morel, H., Calabuig, E., et al. (2018). An outbreak due to Candida auris with prolonged colonisation and candidaemia in a tertiary care European hospital. Mycoses 61, 498-505. doi: 10.1111/myc. 12781

Ruiz-Gaitán, A. C., Cantón, E., Fernández-Rivero, M. E., Ramírez, P., and Pemán, J. (2019). Outbreak of Candida auris in Spain: a comparison of antifungal activity by three methods with published data. Int. J. Antimicrob. Agents 53, 541-546. doi: 10.1016/j.ijantimicag.2019.02.005

Rybak, J. M., Doorley, L. A., Nishimoto, A. T., Barker, K. S., Palmer, G. E., and Rogers, P. D. (2019). Abrogation of triazole resistance upon deletion of CDR1 in a clinical Isolate of Candida auris. Antimicrob. Agents Chemother. 63:e00057-19. doi: 10.1128/AAC.00057-19

Saluja, P., and Prasad, G. S. (2008). Candida ruelliae sp. nov., a novel yeast species isolated from flowers of Ruellia sp. (Acanthaceae). FEMS Yeast Res. 8, 660-666. doi: 10.1111/j.1567-1364.2008.00372.x

Santos, M. A. S., Gomes, A. C., Santos, M. C., Carreto, L. C., and Moura, G. R. (2011). The genetic code of the fungal CTG clade. C. R. Biol. 334, 607-611. doi: 10.1016/j.crvi.2011.05.008

Sarma, S., and Upadhyay, S. (2017). Current perspective on emergence, diagnosis and drug resistance in Candida auris. Infect. Drug Resist. 10, 155-165. doi: 10.2147/IDR.S116229

Satoh, K., Makimura, K., Hasumi, Y., Nishiyama, Y., Uchida, K., and Yamaguchi, H. (2009). Candida auris sp. nov., a novel ascomycetous yeast isolated from the external ear canal of an inpatient in a Japanese hospital. Microbiol. Immunol. 53, 41-44. doi: 10.1111/j.1348-0421.2008.00083.x
Schelenz, S., Hagen, F., Rhodes, J. L., Abdolrasouli, A., Chowdhary, A., Hall, A., et al. (2016). First hospital outbreak of the globally emerging Candida auris in a European hospital. Antimicrob. Resist. Infect. Control 5:35. doi: 10.1186/s13756016-0132-5

Schwartz, I. S., and Hammond, G. W. (2017). First reported case of multidrugresistant Candida auris in Canada. Can. Commun. Dis. Rep. 43, 150-153. doi: 10.14745/ccdr.v43i78a02

Shahana, S., Childers, D. S., Ballou, E. R., Bohovych, I., Odds, F. C., Gow, N. A. R., et al. (2014). New Clox systems for rapid and efficient gene disruption in Candida albicans. PLoS One 9:e100390. doi: 10.1371/journal.pone.010 0390

Sherry, L., Ramage, G., Kean, R., Borman, A., Johnson, E. M., Richardson, M. D., et al. (2017). Biofilm-forming capability of highly virulent, multidrug-resistant Candida auris. Emerg. Infect. Dis. 23, 328-331. doi: 10.3201/eid2302.161320

Singh, S., Uppuluri, P., Mamouei, Z., Alqarihi, A., Elhassan, H., French, S., et al. (2019). The NDV-3A vaccine protects mice from multidrug resistant Candida auris infection. PLoS Pathog. 15:e1007460. doi: 10.1371/journal.ppat.1007460

Sucher, A. J., Chahine, E. B., and Balcer, H. E. (2009). Echinocandins: the newest class of antifungals. Ann. Pharmacother. 43, 1647-1657. doi: 10.1345/aph. $1 \mathrm{M} 237$

Sugita, T., Takashima, M., Poonwan, N., and Mekha, N. (2006). Candida pseudohaemulonii sp. nov., an amphotericin B-and azole-resistant yeast species, isolated from the blood of a patient from Thailand. Microbiol. Immunol. 50, 469-473. doi: 10.1111/j.1348-0421.2006.tb03816.x

Taff, H. T., Mitchell, K. F., Edward, J. A., and Andes, D. R. (2013). Mechanisms of Candida biofilm drug resistance. Future Microbiol. 8, 1325-1337. doi: 10.2217/ fmb.13.101

Tan, Y. E., and Tan, A. L. (2018). Arrival of Candida auris fungus in Singapore: report of the first 3 Cases. Ann. Acad. Med. Singapore 47, 260-262.

Tian, S., Rong, C., Nian, H., Li, F., Chu, Y., Cheng, S., et al. (2018). First cases and risk factors of super yeast Candida auris infection or colonization from Shenyang, China. Emerg. Microbes Infect. 7:128. doi: 10.1038/s41426-0180131-0

Toda, M., Williams, S. R., Berkow, E. L., Farley, M. M., Harrison, L. H., Bonner, L., et al. (2019). Population-based active surveillance for culture-confirmed candidemia - four sites, United States, 2012-2016. MMWR Surveill. Summ. 68, 1-15. doi: 10.15585/mmwr.ss6808al

Tracking Candida auris | CDC (2020). Available online at: https://www.cdc.gov/ fungal/candida-auris/tracking-c-auris.html (Accessed March 4, 2020). doi: 10. 15585/mmwr.ss6808a1

Tsui, C., Kong, E. F., and Jabra-Rizk, M. A. (2016). Pathogenesis of Candida albicans biofilm. Pathog. Dis. 74:ftw018. doi: 10.1093/femspd/ftw018

van Schalkwyk, E., Mpembe, R. S., Thomas, J., Shuping, L., Ismail, H., Lowman, W., et al. (2019). Epidemiologic shift in candidemia driven by Candida auris, South Africa, 2016-2017. Emerg. Infect. Dis. 25, 1698-1707. doi: 10.3201/ eid2509.190040

van Uden, and Kolipinski, M. C. (1962). Torulopsis haemulonii nov. spec., a yeast from the Atlantic Ocean. Antonie Van Leeuwenhoek 28, 78-80. doi: 10.1007/ bf02538724

Vatanshenassan, M., Boekhout, T., Meis, J. F., Berman, J., Chowdhary, A., BenAmi, R., et al. (2019). Candida auris identification and rapid antifungal susceptibility testing against echinocandins by MALDI-TOF MS. Front. Cell. Infect. Microbiol. 9:20. doi: 10.3389/fcimb.2019.00020

Vermes, A., Guchelaar, H. J., and Dankert, J. (2000). Flucytosine: a review of its pharmacology, clinical indications, pharmacokinetics, toxicity and drug interactions. J. Antimicrob. Chemother. 46, 171-179. doi: 10.1093/jac/46.2.171

Vogelzang, E. H., Weersink, A. J. L., van Mansfeld, R., Chow, N. A., Meis, J. F., and van Dijk, K. (2019). The first two cases of Candida auris in The Netherlands. J. Fungi 5:91. doi: 10.3390/jof5040091

Wang, S.-A., Jia, J.-H., and Bai, F.-Y. (2008). Candida alocasiicola sp. nov., Candida hainanensis sp. nov., Candida heveicola sp. nov. and Candida musiphila sp. nov., novel anamorphic, ascomycetous yeast species isolated from plants. Antonie Van Leeuwenhoek 94, 257-265. doi: 10.1007/s10482-0089238-y

Wang, X., Bing, J., Zheng, Q., Zhang, F., Liu, J., Yue, H., et al. (2018). The first isolate of Candida auris in China: clinical and biological aspects. Emerg. Microbes Infect. 7:93. doi: 10.1038/s41426-018-0095-0 
Warris, A. (2018). Candida auris, what do paediatricians need to know? Arch. Dis. Child. 103, 891-894. doi: 10.1136/archdischild-2017-313960

Wasi, M., Khandelwal, N. K., Moorhouse, A. J., Nair, R., Vishwakarma, P., Bravo Ruiz, G., et al. (2019). ABC transporter genes show upregulated expression in drug-resistant clinical isolates of Candida auris: a genomewide characterization of ATP-Binding Cassette (ABC) transporter genes. Front. Microbiol. 10:1445. doi: 10.3389/fmicb.2019.01445

Welsh, R. M., Bentz, M. L., Shams, A., Houston, H., Lyons, A., Rose, L. J., et al. (2017). survival, persistence, and isolation of the emerging multidrugresistant pathogenic yeast Candida auris on a plastic health care surface. J. Clin. Microbiol. 55, 2996-3005. doi: 10.1128/JCM.00921-17

Whaley, S. G., Berkow, E. L., Rybak, J. M., Nishimoto, A. T., Barker, K. S., and Rogers, P. D. (2017). Azole antifungal resistance in Candida albicans and emerging non-albicans Candida Species. Front. Microbiol. 7:2173. doi: 10.3389/ fmicb.2016.02173

Wiederhold, N. P., Lockhart, S. R., Najvar, L. K., Berkow, E. L., Jaramillo, R., Olivo, M., et al. (2019). The fungal Cyp51-specific inhibitor VT-1598 demonstrates in vitro and in vivo activity against Candida auris. Antimicrob. Agents Chemother. 63:e02233-18. doi: 10.1128/AAC.02233-18

Yue, H., Bing, J., Zheng, Q., Zhang, Y., Hu, T., Du, H., et al. (2018). Filamentation in Candida auris, an emerging fungal pathogen of humans: passage through the mammalian body induces a heritable phenotypic switch. Emerg. Microbes Infect. 7:188. doi: 10.1038/s41426-018-0187-x
Zamith-Miranda, D., Heyman, H. M., Cleare, L. G., Couvillion, S. P., Clair, G. C., Bredeweg, E. L., et al. (2019). Multi-omics signature of Candida auris, an emerging and multidrug-resistant pathogen. mSystems 4:e00257-19. doi: 10. 1128/mSystems.00257-19

Zhao, X., Oh, S.-H., Yeater, K. M., and Hoyer, L. L. (2005). Analysis of the Candida albicans Als2 $\mathrm{p}$ and Als4p adhesins suggests the potential for compensatory function within the Als family. Microbiol. Read. Engl. 151, 1619-1630. doi: 10.1099/mic.0.27763-0

Zheng, X., Wang, Y., and Wang, Y. (2004). Hgcl, a novel hypha-specific G1 cyclinrelated protein regulates Candida albicans hyphal morphogenesis. EMBO J. 23, 1845-1856. doi: 10.1038/sj.emboj.7600195

Conflict of Interest: The authors declare that the research was conducted in the absence of any commercial or financial relationships that could be construed as a potential conflict of interest.

Copyright (C) 2020 Chybowska, Childers and Farrer. This is an open-access article distributed under the terms of the Creative Commons Attribution License (CC BY). The use, distribution or reproduction in other forums is permitted, provided the original author(s) and the copyright owner(s) are credited and that the original publication in this journal is cited, in accordance with accepted academic practice. No use, distribution or reproduction is permitted which does not comply with these terms. 